\title{
Enhancement of the quadrupole interaction of an atom with the guided light of an ultrathin optical fiber
}

\author{
Fam Le Kien, ${ }^{1}$ Tridib Ray, ${ }^{2}$ Thomas Nieddu, ${ }^{2}$ Thomas Busch, ${ }^{1}$ and Síle Nic Chormaic ${ }^{2,3}$ \\ ${ }^{1}$ Quantum Systems Unit, Okinawa Institute of Science and Technology Graduate University, Onna, Okinawa 904-0495, Japan \\ ${ }^{2}$ Light-Matter Interactions Unit, Okinawa Institute of Science and Technology Graduate University, Onna, Okinawa 904-0495, Japan \\ ${ }^{3}$ School of Chemistry and Physics, University of KwaZulu-Natal, Durban, KwaZulu-Natal 4001, South Africa
}

(Received 19 September 2017; published 16 January 2018)

\begin{abstract}
We investigate the electric quadrupole interaction of an alkali-metal atom with guided light in the fundamental and higher-order modes of a vacuum-clad ultrathin optical fiber. We calculate the quadrupole Rabi frequency, the quadrupole oscillator strength, and their enhancement factors. In the example of $\mathrm{a}^{87} \mathrm{Rb}$ atom, we study the dependencies of the quadrupole Rabi frequency on the quantum numbers of the transition, the mode type, the phase circulation direction, the propagation direction, the orientation of the quantization axis, the position of the atom, and the fiber radius. We find that the root-mean-square (rms) quadrupole Rabi frequency reduces quickly but the quadrupole oscillator strength varies slowly with increasing radial distance. We show that the enhancement factors of the rms Rabi frequency and the oscillator strength do not depend on any characteristics of the internal atomic states except for the atomic transition frequency. The enhancement factor of the oscillator strength can be significant even when the atom is far away from the fiber. We show that, in the case where the atom is positioned on the fiber surface, the oscillator strength for the quasicircularly polarized fundamental mode $\mathrm{HE}_{11}$ has a local minimum at the fiber radius $a \simeq 107 \mathrm{~nm}$, and is larger than that for quasicircularly polarized higher-order hybrid modes, transverse electric modes, and transverse magnetic modes in the region $a<498.2 \mathrm{~nm}$.
\end{abstract}

DOI: 10.1103/PhysRevA.97.013821

\section{INTRODUCTION}

Dipole-allowed optical transitions in atoms, ions, and molecules play a key role in modern atomic, molecular, and optical physics [1]. The corresponding Rabi frequency is linearly proportional to the amplitude of the light field. Energy levels that are not connected to lower energy levels by dipole-allowed transitions are metastable states and have many applications ranging from precision clocks [2] to quantum gates [3]. Electric quadrupole transitions, on the other hand, are proportional to the gradient of the electric field and are less explored. Techniques to investigate nondipole transitions have been explored theoretically and experimentally for atoms in free space [4-17], in evanescent fields [18-20], near a dielectric microsphere [21], near an ideally conducting cylinder [22], and near plasmonic nanostructures $[23,24]$. However, the difficulty in achieving large electric field gradients over a long distance makes the study of quadrupole transitions in an extended medium a challenging task.

Ultrathin optical fibers [25-27] allow tightly radially confined light to propagate over a long distance. Apart from a high intensity, the evanescent field that extends radially beyond the physical boundary of an ultrathin fiber also offers a large intensity gradient in the radial direction $[28,29]$. The

Published by the American Physical Society under the terms of the Creative Commons Attribution 4.0 International license. Further distribution of this work must maintain attribution to the author(s) and the published article's title, journal citation, and DOI. corresponding intensity gradient can be used to confine atoms near the surface of an ultrathin fiber [30-32]. Furthermore, the higher-order modes of an ultrathin fiber [33-35] may also offer an azimuthal phase gradient.

The aim of the present paper is to investigate the electric quadrupole interaction of an alkali-metal atom with guided light in the fundamental and higher-order modes of a vacuumclad ultrathin optical fiber. We calculate the quadrupole Rabi frequency, the quadrupole oscillator strength, and their enhancement factors. In the example of $\mathrm{a}^{87} \mathrm{Rb}$ atom, we study the dependencies of these characteristics on the quantum numbers of the transition, the mode type, the phase circulation direction, the propagation direction, the orientation of the quantization axis, the position of the atom, and the fiber radius.

The paper is organized as follows. In Sec. II we study the electric quadrupole interaction of an alkali-metal atom with an arbitrary monochromatic light field. In Sec. III we examine the interaction of the atom with guided light of an ultrathin optical fiber and derive an expression for the enhancement factor of the quadrupole oscillator strength in terms of the fiber mode functions. In Sec. IV we present numerical results. Our conclusions are given in Sec. V.

\section{QUADRUPOLE INTERACTION OF AN ATOM WITH AN ARBITRARY LIGHT FIELD}

Consider an atom with a single valence electron interacting with an external optical field $\mathbf{E}$ through an electric quadrupole transition. We use Cartesian coordinates $\left\{x_{1}, x_{2}, x_{3}\right\}$ to describe the electric quadrupole and the internal states of the atom [see Fig. 1(a)]. We assume that the center of mass of the 
(a)

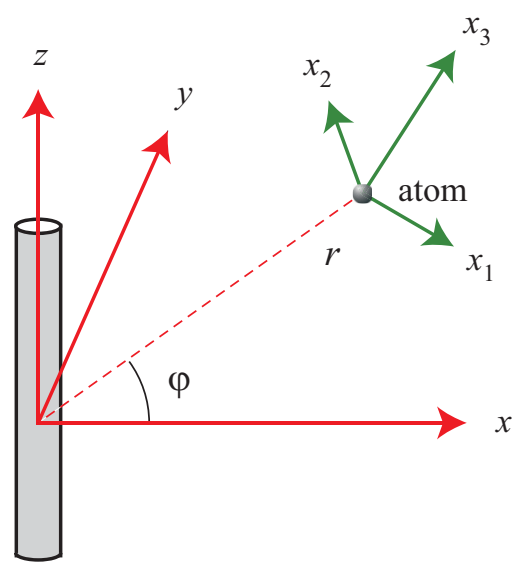

ultrathin fiber

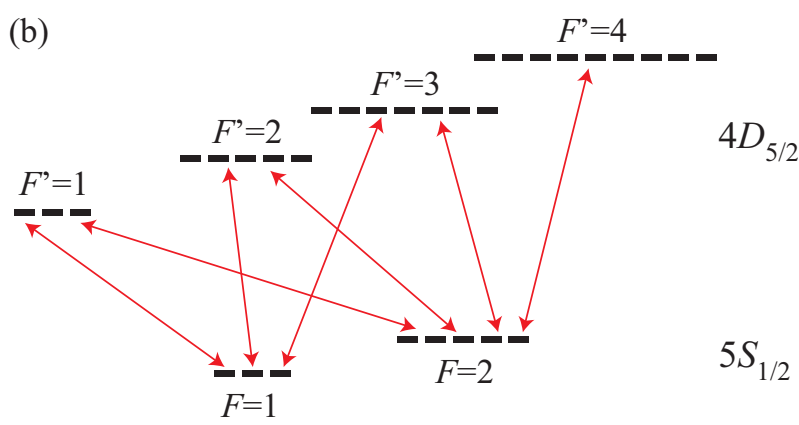

FIG. 1. (a) Atom with the local quantization coordinate system $\left\{x_{1}, x_{2}, x_{3}\right\}$ in the vicinity of an ultrathin optical fiber with the fiberbased Cartesian coordinate system $\{x, y, z\}$ and the corresponding cylindrical coordinate system $\{r, \varphi, z\}$. (b) Schematic of the hyperfinestructure (hfs) levels of the $4 D_{5 / 2}$ and $5 S_{1 / 2}$ states of a ${ }^{87} \mathrm{Rb}$ atom.

atom is located at the origin $\mathbf{x}=0$ of this coordinate system. The electric quadrupole moment tensor $Q_{i j}$ of the atom, with $i, j=1,2,3$, is defined as

$$
Q_{i j}=e\left(3 x_{i} x_{j}-R^{2} \delta_{i j}\right),
$$

where $x_{i}$ is the $i$ th coordinate of the valence electron of the atom and $R=\sqrt{x_{1}^{2}+x_{2}^{2}+x_{3}^{2}}$ is the distance from the electron to the center of mass of the atom. The electric quadrupole interaction energy is [36]

$$
W=-\left.\frac{1}{6} \sum_{i j} Q_{i j} \frac{\partial E_{j}}{\partial x_{i}}\right|_{\mathbf{x}=0},
$$

where the spatial derivatives of the field components $E_{j}$ with respect to the coordinates $x_{i}$ are evaluated at the position $\mathbf{x}=0$ of the atom.

We represent the field as $\mathbf{E}=\left(\mathcal{E} e^{-i \omega t}+\mathcal{E}^{*} e^{i \omega t}\right) / 2$, where $\mathcal{E}$ is the field amplitude and $\omega$ the field frequency. Let $|e\rangle$ and $|g\rangle$ be upper and lower states of the atom, with energies $\hbar \omega_{e}$ and $\hbar \omega_{g}$, respectively. In the interaction picture and the rotating-wave approximation, the interaction Hamiltonian of the system can be written as

$$
H_{I}=-\frac{\hbar}{2} \sum_{e g} \Omega_{g e} e^{-i\left(\omega-\omega_{e g}\right) t} \sigma_{e g}+\text { H.c. }
$$

where $\omega_{e g}=\omega_{e}-\omega_{g}$ is the atomic transition frequency and

$$
\Omega_{g e}=\frac{1}{6 \hbar} \sum_{i j}\left\langle e\left|Q_{i j}\right| g\right\rangle \frac{\partial \mathcal{E}_{j}}{\partial x_{i}}
$$

is the Rabi frequency for the quadrupole transition between the states $|g\rangle$ and $|e\rangle$.

Consider the case of an alkali-metal atom with degenerate transitions between the magnetic sublevels $|g\rangle=|n F M\rangle$ and $|e\rangle=\left|n^{\prime} F^{\prime} M^{\prime}\right\rangle$ [see Fig. 1(b)]. Here, $n$ denotes the principal quantum number and also all additional quantum numbers not shown explicitly, $F$ is the quantum number for the total angular momentum of the atom, and $M$ is the magnetic quantum number. The matrix elements $\left\langle n^{\prime} F^{\prime} M^{\prime}\left|Q_{i j}\right| n F M\right\rangle$ of the quadrupole tensor operators $Q_{i j}$ are, as shown in Appendix A, given as [7]

$$
\begin{aligned}
& \left\langle n^{\prime} F^{\prime} M^{\prime}\left|Q_{i j}\right| n F M\right\rangle \\
& =3 e u_{i j}^{\left(M^{\prime}-M\right)}(-1)^{F^{\prime}-M^{\prime}}\left(\begin{array}{ccc}
F^{\prime} & 2 & F \\
-M^{\prime} & M^{\prime}-M & M
\end{array}\right) \\
& \quad \times\left\langle n^{\prime} F^{\prime}\left\|T^{(2)}\right\| n F\right\rangle,
\end{aligned}
$$

where the matrices $u_{i j}^{(q)}$ with $q=-2,-1,0,1,2$ are given by Eqs. (A12), the array in the parentheses is a $3 j$ symbol, and the invariant factor $\left\langle n^{\prime} F^{\prime}\left\|T^{(2)}\right\| n F\right\rangle$ is the reduced matrix element of the tensor operators $T_{q}^{(2)}=2(2 \pi / 15)^{1 / 2} R^{2} Y_{2 q}(\vartheta, \phi)$. Here, $Y_{l q}$ is a spherical harmonic function of degree $l$ and order $q$, and $\vartheta$ and $\phi$ are spherical angles in the spherical coordinates $\{R, \vartheta, \phi\}$ associated with the Cartesian coordinates $\left\{x_{1}, x_{2}, x_{3}\right\}$.

It is clear from Eq. (5) that the electric quadrupole transition selection rules for $F$ and $F^{\prime}$ and for $M$ and $M^{\prime}$ are $\left|F^{\prime}-F\right| \leqslant$ $2 \leqslant F^{\prime}+F$ and $\left|M^{\prime}-M\right| \leqslant 2$. We note that the selection rules for the quantum numbers $J$ and $J^{\prime}$ of the total angular momenta of the electrons are $\left|J^{\prime}-J\right| \leqslant 2 \leqslant J^{\prime}+J$. We also note that the selection rules for the quantum numbers $L$ and $L^{\prime}$ of the total orbital angular momenta of the electrons are $\left|L^{\prime}-L\right|=0,2$ and $L^{\prime}+L \geqslant 2$.

We now calculate the quadrupole Rabi frequency $\Omega_{g e}=$ $\Omega_{F M F^{\prime} M^{\prime}}$, defined by Eq. (4). When we insert Eq. (5) into Eq. (4), we obtain

$$
\begin{aligned}
\Omega_{F M F^{\prime} M^{\prime}}= & \frac{e}{2 \hbar}(-1)^{F^{\prime}-M^{\prime}}\left(\begin{array}{ccc}
F^{\prime} & 2 & F \\
-M^{\prime} & M^{\prime}-M & M
\end{array}\right) \\
& \times\left\langle n^{\prime} F^{\prime}\left\|T^{(2)}\right\| n F\right\rangle \sum_{i j} u_{i j}^{\left(M^{\prime}-M\right)} \frac{\partial \mathcal{E}_{j}}{\partial x_{i}} .
\end{aligned}
$$

In general, the Rabi frequency $\Omega_{F M F^{\prime} M^{\prime}}$ for the transition between the atomic states $|n F M\rangle$ and $\left|n^{\prime} F^{\prime} M^{\prime}\right\rangle$ depends on the relative orientation of the quantization axis $x_{3}$ with respect to the electric field vector $\mathcal{E}$.

The root-mean-square (rms) Rabi frequency $\bar{\Omega}_{F F^{\prime}}$ is given by the rule [37]

$$
\bar{\Omega}_{F F^{\prime}}^{2}=\sum_{M M^{\prime}}\left|\Omega_{F M F^{\prime} M^{\prime}}\right|^{2} .
$$

We insert Eq. (6) into Eq. (7) and perform the summations over $M$ and $M^{\prime}$. Then, we obtain

$$
\bar{\Omega}_{F F^{\prime}}^{2}=\frac{e^{2}}{20 \hbar^{2}}\left|\left\langle n^{\prime} F^{\prime}\left\|T^{(2)}\right\| n F\right\rangle\right|^{2} \sum_{q}\left|\sum_{i j} u_{i j}^{(q)} \frac{\partial \mathcal{E}_{j}}{\partial x_{i}}\right|^{2} .
$$


We note that Eqs. (6) and (8) can be used for a monochromatic light field with an arbitrary space-dependent amplitude $\mathcal{E}$. In the particular case of standing-wave laser fields, Eqs. (6) and (8) reduce to the results of Ref. [7].

We assume that the field is near to resonance with the atom, that is, $\omega \simeq \omega_{0}$, where $\omega_{0} \equiv \omega_{e g}$. The oscillator strength $f_{F F^{\prime}}$ can be calculated from the rms Rabi frequency $\bar{\Omega}_{F F^{\prime}}$ by using the relation [37]

$$
\bar{\Omega}_{F F^{\prime}}^{2}=\frac{e^{2}|\mathcal{E}|^{2}}{2 \hbar m_{e} \omega_{0}}(2 F+1) f_{F F^{\prime}},
$$

where $m_{e}$ is the mass of an electron. This yields

$$
\begin{aligned}
f_{F F^{\prime}}= & \frac{m_{e} \omega_{0}}{18 \hbar e^{2}(2 F+1)} \\
& \times \sum_{M M^{\prime}}\left|\sum_{i j}\left\langle n^{\prime} F^{\prime} M^{\prime}\left|Q_{i j}\right| n F M\right\rangle \frac{1}{\mathcal{E}} \frac{\partial \mathcal{E}_{j}}{\partial x_{i}}\right|^{2} .
\end{aligned}
$$

Equation (10) can be used for a monochromatic light field with an arbitrary space-dependent amplitude $\mathcal{E}$. In the particular case where $\mathcal{E}=\mathcal{E}_{0} e^{i \mathbf{K} \cdot \mathbf{x}}$ with $\mathcal{E}_{0}$ and $\mathbf{K}$ being constant real or complex vectors, Eq. (10) reduces to an expression that is in agreement with Refs. [18-20].

With the help of Eqs. (8) and (9), we find

$$
f_{F F^{\prime}}=\frac{m_{e} \omega_{0}}{10 \hbar} \frac{\left|\left\langle n^{\prime} F^{\prime}\left\|T^{(2)}\right\| n F\right\rangle\right|^{2}}{2 F+1} \sum_{q}\left|\sum_{i j} u_{i j}^{(q)} \frac{1}{\mathcal{E}} \frac{\partial \mathcal{E}_{j}}{\partial x_{i}}\right|^{2} \text {. }
$$

Like Eq. (10), Eq. (11) can be used for an arbitrary monochromatic light field. Due to the summation over $M$ and $M^{\prime}$ in Eq. (7), the rms Rabi frequency $\bar{\Omega}_{F F^{\prime}}$ and, consequently, the oscillator strength $f_{F F^{\prime}}$ do not depend on the orientation of the quantization axis $x_{3}$. The quadrupole oscillator strength $f_{F F^{\prime}}$, given by Eq. (11), is a measure that characterizes the proportionality of the rms Rabi frequency $\bar{\Omega}_{F F^{\prime}}$ to the field amplitude magnitude $|\mathcal{E}|$ through Eq. (9). The measure $f_{F F^{\prime}}$ depends on not only the reduced quadrupole matrix element $\left\langle n^{\prime} F^{\prime}\left\|T^{(2)}\right\| n F\right\rangle$ of the atom but also the ratios between the field gradients $\partial \mathcal{E}_{j} / \partial x_{i}$ and the field amplitude $\mathcal{E}$. It is clear that the oscillator strength $f_{F F^{\prime}}$ is a measure per unit intensity. We note that, for atoms in free space, the oscillator strength can be interpreted as the ratio between the quantum-mechanical transition rate and the classical absorption rate of a singleelectron oscillator with the same frequency $[36,37]$. However, this interpretation may not be valid for atoms in the vicinity of an object because the modifications of the transition rate are much more complicated than that of the Rabi frequency.

We introduce the notations $\bar{\Omega}_{F F^{\prime}}^{(0)}$ and $f_{F F^{\prime}}^{(0)}$ for the rms Rabi frequency and oscillator strength of an atom interacting with a plane-wave light field in free space via an electric quadrupole transition. According to $[6,7,20]$ and Appendix B, we have

$$
\bar{\Omega}_{F F^{\prime}}^{(0) 2}=\frac{e^{2} k^{2}|\mathcal{E}|^{2}}{40 \hbar^{2}}\left|\left\langle n^{\prime} F^{\prime}\left\|T^{(2)}\right\| n F\right\rangle\right|^{2}
$$

and

$$
f_{F F^{\prime}}^{(0)}=\frac{m_{e} \omega_{0}^{3}}{20 \hbar c^{2}} \frac{\left|\left\langle n^{\prime} F^{\prime}\left\|T^{(2)}\right\| n F\right\rangle\right|^{2}}{2 F+1} .
$$

The enhancements of the rms Rabi frequency and oscillator strength in arbitrary light are characterized by the factors

$$
\begin{gathered}
\eta_{\text {Rabi }}=\frac{\bar{\Omega}_{F F^{\prime}}}{\bar{\Omega}_{F F^{\prime}}^{(0)}}, \\
\eta_{\text {osc }}=\frac{f_{F F^{\prime}}}{f_{F F^{\prime}}^{(0)}} .
\end{gathered}
$$

We find

$$
\eta_{\text {osc }}=\eta_{\text {Rabi }}^{2}=\frac{2}{k_{0}^{2}|\mathcal{E}|^{2}} \sum_{q}\left|\sum_{i j} u_{i j}^{(q)} \frac{\partial \mathcal{E}_{j}}{\partial x_{i}}\right|^{2} .
$$

According to expressions (8) and (11), the dependencies of $\bar{\Omega}_{F F^{\prime}}^{2}$ and $f_{F F^{\prime}}$ on $F$ and $F^{\prime}$ are included only in the factors $\left|\left\langle n^{\prime} F^{\prime}\left\|T^{(2)}\right\| n F\right\rangle\right|^{2}$ and $\left|\left\langle n^{\prime} F^{\prime}\left\|T^{(2)}\right\| n F\right\rangle\right|^{2} /(2 F+1)$. These scaling factors are determined by the internal atomic states. They do not depend on the position of the atom and the radius of the fiber. Consequently, the shapes of the dependencies of $\bar{\Omega}_{F F^{\prime}}^{2}$ and $f_{F F^{\prime}}$ on the position of the atom and the radius of the fiber do not depend on the quantum numbers $F$ and $F^{\prime}$. Meanwhile, Eq. (15) shows that the magnitudes of the enhancement factors $\eta_{\text {Rabi }}$ and $\eta_{\text {osc }}$ do not depend on $F$ and $F^{\prime}$. Moreover, these enhancement factors do not depend on any characteristics of the atomic states except for the atomic transition frequency $\omega_{0}$. They are determined by the ratios between the gradients $\partial \mathcal{E}_{j} / \partial x_{i}$ and the amplitude $\mathcal{E}$ of the field operating at the atomic resonant frequency $\omega_{0}$.

We note that the oscillator strength $f_{J J^{\prime}}$ of the transition from a lower fine-structure level $|n J\rangle$ to an upper fine-structure level $\left|n^{\prime} J^{\prime}\right\rangle$ of the atom may be obtained by summing up $f_{F F^{\prime}}$ over all values of $F^{\prime}$. The result is

$$
f_{J J^{\prime}}=\frac{m_{e} \omega_{0}}{10 \hbar} \frac{\left|\left\langle n^{\prime} J^{\prime}\left\|T^{(2)}\right\| n J\right\rangle\right|^{2}}{2 J+1} \sum_{q}\left|\sum_{i j} u_{i j}^{(q)} \frac{1}{\mathcal{E}} \frac{\partial \mathcal{E}_{j}}{\partial x_{i}}\right|^{2} \text {. }
$$

In the case of an atom interacting with a plane-wave light field in free space, we have $[6,7,20]$

$$
f_{J J^{\prime}}^{(0)}=\frac{m_{e} \omega_{0}^{3}}{20 \hbar c^{2}} \frac{\left|\left\langle n^{\prime} J^{\prime}\left\|T^{(2)}\right\| n J\right\rangle\right|^{2}}{2 J+1} .
$$

The relation between $f_{F F^{\prime}}$ and $f_{J J^{\prime}}$ is $[18,19,38]$

$$
f_{F F^{\prime}}=\left(2 F^{\prime}+1\right)(2 J+1)\left\{\begin{array}{ccc}
F^{\prime} & 2 & F \\
J & I & J^{\prime}
\end{array}\right\}^{2} f_{J J^{\prime}},
$$

where $I$ is the nuclear spin quantum number and the array in the curly braces is a $6 j$ symbol.

\section{QUADRUPOLE INTERACTION OF AN ATOM WITH GUIDED LIGHT}

We consider the electric quadrupole interaction between the atom and a guided light field of a vacuum-clad ultrathin optical fiber [see Fig. 1(a)]. We assume that the fiber is a dielectric cylinder of radius $a$ and refractive index $n_{1}$ and is surrounded by an infinite background medium of refractive index $n_{2}$, where $n_{2}<n_{1}$. We consider the case where the refractive indices $n_{1}$ and $n_{2}$ are real. We use Cartesian coordinates $\{x, y, z\}$, where $z$ is the coordinate along the fiber axis, and also cylindrical coordinates $\{r, \varphi, z\}$, where $r$ and $\varphi$ are the polar coordinates in the fiber transverse plane $x y$. 
We note that our model consists of a fiber and a single atom. In the case where there are many atoms deposited on the surface of the fiber, due to the effect of the coverage on the mode structure of the field, our model is not valid. We also note that, when the atom is very close to the surface, the energy levels and the transition rates of the atom are modified. The shifts of the energy levels and the changes in the transition rates are related to each other by the Kramers-Kronig relation. In addition, the wave functions of the internal states of the atom are also modified. Such changes in the wave functions may induce a nonzero dipole matrix element for a transition that is dipole-forbidden in free space. Since the optical transition frequency is huge, the effect of the surface-induced energy level shift on the transition rate can be neglected when the atom is not too close to the surface (when the distance is larger than a few nanometers in the case of a flat silica surface) [39]. On the other hand, the modification of the atomic state wave functions is also a weak effect that can occur only when the atom is very close to the surface. Therefore, for simplicity, we neglect the effect of the fiber on the atomic energy levels and wave functions. We also neglect other effects such as material absorption, nonradiative decay, surface roughness, short-range repulsion, and adsorption. Because of these approximations, the results for the case where the atom is effectively placed on the fiber surface are just the limiting values for the case where the atom is close enough but not too close to the surface so that the approximations are valid. When the atom is close enough to the surface, due to various effects that are not taken into account in our treatment, our results are not valid.

We assume that the fiber supports the fundamental $\mathrm{HE}_{11}$ mode and a few higher-order modes [40] in a finite bandwidth around the central frequency $\omega_{0}=\omega_{e}-\omega_{g}$ of the atom. The theory of fiber guided modes is given in Ref. [40] and is summarized in Appendix C. The propagation constant $\beta$ of a guided mode is determined by Eq. (C1). A guided mode in the class of quasicircularly polarized hybrid modes, transverse electric modes, and transverse magnetic modes can be labeled by an index $\mu=(\omega, N, f, p)$. Here, $\omega$ is the mode frequency, the notation $N=\mathrm{HE}_{l m}, \mathrm{EH}_{l m}, \mathrm{TE}_{0 m}$, or $\mathrm{TM}_{0 m}$ stands for the mode type, with $l=1,2, \ldots$ and $m=1,2, \ldots$ being the azimuthal and radial mode orders, respectively, the index $f=+1$ or -1 denotes respectively the forward or backward propagation direction along the fiber axis $z$, and $p$ is the polarization index. The $\mathrm{HE}_{l m}$ and $\mathrm{EH}_{l m}$ modes are hybrid modes. For these modes, the azimuthal order is $l \neq 0$, and the index $p$ is equal to +1 or -1 , indicating the counterclockwise or clockwise circulation direction of the helical phase front. The $\mathrm{TE}_{0 m}$ and $\mathrm{TM}_{0 m}$ modes are transverse electric and magnetic modes. For these modes, the azimuthal mode order is $l=0$, the mode polarization is single, and the polarization index $p$ can be dropped.

For a quasicircularly polarized hybrid $\mathrm{HE}_{l m}$ or $\mathrm{EH}_{l m}$ mode with the propagation direction $f$ and the phase circulation direction $p$, the field amplitude is $[34,40]$

$$
\mathcal{E}=\left(e_{r} \hat{\mathbf{r}}+p e_{\varphi} \hat{\boldsymbol{\varphi}}+f e_{z} \hat{\mathbf{z}}\right) e^{i f \beta z+i p l \varphi},
$$

where $e_{r}, e_{\varphi}$, and $e_{z}$ are given by Eqs. (C10) and (C11) for $\beta>0$ and $l>0$.
For a $\mathrm{TE}_{0 m}$ mode with the propagation direction $f$, the field amplitude is $[34,40]$

$$
\mathcal{E}=e_{\varphi} \hat{\varphi} e^{i f \beta z},
$$

where the only nonzero cylindrical component $e_{\varphi}$ is given by Eqs. (C12) and (C13).

For a $\mathrm{TM}_{0 m}$ mode with the propagation direction $f$, the field amplitude is $[34,40]$

$$
\mathcal{E}=\left(e_{r} \hat{\mathbf{r}}+f e_{z} \hat{\mathbf{z}}\right) e^{i f \beta z},
$$

where the components $e_{r}$ and $e_{z}$ are given by Eqs. (C14) and (C15) for $\beta>0$. An important property of the mode functions of hybrid and TM modes is that the longitudinal component $e_{z}$ is nonvanishing and in quadrature ( $\pi / 2$ out of phase) with the radial component $e_{r}$.

Quasilinearly polarized hybrid modes are linear superpositions of counterclockwise and clockwise quasicircularly polarized hybrid modes. The amplitude of the guided field in a quasilinearly polarized hybrid mode can be written in the form

$$
\begin{aligned}
\mathcal{E}= & \sqrt{2}\left[\hat{\mathbf{r}} e_{r} \cos \left(l \varphi-\varphi_{\mathrm{pol}}\right)+i \hat{\boldsymbol{\varphi}} e_{\varphi} \sin \left(l \varphi-\varphi_{\mathrm{pol}}\right)\right. \\
& \left.+f \hat{\mathbf{z}} e_{z} \cos \left(l \varphi-\varphi_{\mathrm{pol}}\right)\right] e^{i f \beta z},
\end{aligned}
$$

where the phase angle $\varphi_{\text {pol }}$ determines the orientation of the symmetry axes of the mode profile in the fiber transverse plane. In particular, the specific phase angle values $\varphi_{\text {pol }}=0$ and $\pi / 2$ define two orthogonal polarization profiles, one being symmetric with respect to the $x$ axis and the other being the result of the rotation of the first one by an angle of $\pi / 2 l$ in the fiber transverse plane $x y$.

With the help of some simple coordinate transformations, we can easily calculate the quadrupole Rabi frequency $\Omega_{F M F^{\prime} M^{\prime}}$, the quadrupole oscillator strength $f_{F F^{\prime}}$, and their enhancement factors $\eta_{\text {Rabi }}$ and $\eta_{\text {osc }}$ for an arbitrary guided light field. For quasicircularly polarized hybrid $\mathrm{HE}$ and EH modes, TE modes, and TM modes, a simple analytical expression for the enhancement factor $\eta_{\text {osc }}$ is found to be

$$
\begin{aligned}
\eta_{\mathrm{osc}}= & \frac{1}{k_{0}^{2}|\mathbf{e}|^{2}}\left[\left|e_{r}^{\prime}-\frac{1}{r}\left(i l e_{\varphi}+e_{r}\right)\right|^{2}+\left|e_{\varphi}^{\prime}+\frac{1}{r}\left(i l e_{r}-e_{\varphi}\right)\right|^{2}\right. \\
& +\left|e_{z}^{\prime}+i \beta_{0} e_{r}\right|^{2}+\left|\frac{l}{r} e_{z}+\beta_{0} e_{\varphi}\right|^{2} \\
& \left.+\frac{1}{3}\left|e_{r}^{\prime}-2 i \beta_{0} e_{z}+\frac{1}{r}\left(i l e_{\varphi}+e_{r}\right)\right|^{2}\right] .
\end{aligned}
$$

Here, $e_{r}, e_{\varphi}$, and $e_{z}$ are evaluated at the atomic transition frequency $\omega_{0}$, and the notations $e_{r, \varphi, z}^{\prime}=\partial e_{r, \varphi, z} / \partial r$ and $\beta_{0}=$ $\beta\left(\omega_{0}\right)$ have been introduced. We emphasize that Eq. (23) is not valid for quasilinearly polarized hybrid modes.

We find from Eq. (23) that, in the limit $r \rightarrow \infty$, we have $\eta_{\text {osc }} \rightarrow \eta_{\text {osc }}^{\infty}$, where

$$
\eta_{\mathrm{osc}}^{\infty}= \begin{cases}\frac{q_{0}^{2}+\beta_{0}^{2}}{k_{0}^{2}}+\frac{4 q_{0}^{2} \beta_{0}^{2}}{k_{0}^{2}\left(q_{0}^{2}+\beta_{0}^{2}+\beta_{0}^{2} s_{0}^{2}\right)} & \text { for HE and EH, } \\ \frac{q_{0}^{2}+\beta_{0}^{2}}{k_{0}^{2}} & \text { for TE }, \\ \frac{q_{0}^{2}+\beta_{0}^{2}}{k_{0}^{2}}+\frac{4 q_{0}^{2} \beta_{0}^{2}}{k_{0}^{2}\left(q_{0}^{2}+\beta_{0}^{2}\right)} & \text { for TM. }\end{cases}
$$

Here, $q_{0}$ and $s_{0}$ are the fiber parameters given in Appendix C and are evaluated at the frequency $\omega_{0}$. It is clear that $\eta_{\mathrm{osc}}^{\infty}$ are 
determined by the fiber parameters. Since $\beta_{0} \geqslant k_{0}$, we have $\eta_{\text {osc }}^{\infty} \geqslant 1$.

\section{NUMERICAL RESULTS}

In this section, we demonstrate the results of numerical calculations for the characteristics of an electric quadrupole transition of an atom interacting with a guided light field of an ultrathin optical fiber. As an example, we study the electric quadrupole transition between the ground state $5 S_{1 / 2}$ and the excited state $4 D_{5 / 2}$ of a ${ }^{87} \mathrm{Rb}$ atom. For this transition, we have $L^{\prime}=2, J^{\prime}=5 / 2, L=0, J=1 / 2$, and $I=3 / 2$. The wavelength of the transition is $\lambda_{0}=516.5 \mathrm{~nm}$. The experimentally measured oscillator strength of the transition $5 S_{1 / 2} \rightarrow 4 D_{5 / 2}$ in free space is $f_{J J^{\prime}}^{(0)}=8.06 \times 10^{-7}$ [5]. In our numerical calculations, we assume that the field is at exact resonance with the atom $\left(\omega=\omega_{0}\right)$.

Before we proceed, we note that we choose the quadrupole transition $5 S_{1 / 2} \rightarrow 4 D_{5 / 2}$ of a ${ }^{87} \mathrm{Rb}$ atom to calculate numerically because the oscillator strength of this transition in free space has been measured [5]. Another reason is that a similar transition, namely, the transition $6 S_{1 / 2} \rightarrow 5 D_{5 / 2}$, of cesium atoms in an evanescent field has been studied experimentally [18]. Although a specific quadrupole transition of a specific alkali-metal atom is chosen, our numerical results are relatively general because the enhancement factor $\eta_{\text {osc }}$ of the oscillator strength does not depend on any characteristics of the atomic states except for the atomic transition frequency $\omega_{0}$.

First, we calculate the Rabi frequency $\Omega_{F M F^{\prime} M^{\prime}}$ for the transition between the magnetic sublevels $|F M\rangle$ and $\left|F^{\prime} M^{\prime}\right\rangle$. It is clear from Eq. (6) that $\Omega_{F M F^{\prime} M^{\prime}}$ depends on not only $F$ and $F^{\prime}$ but also $M$ and $M^{\prime}$, and is linearly proportional to the gradient of the amplitude of the light field.

We plot in Fig. 2 the absolute value of the Rabi frequency $\Omega_{F M F^{\prime} M^{\prime}}$ as a function of the radial distance $r$ for different upper sublevels $\left|F^{\prime} M^{\prime}\right\rangle$ and different guided modes $N=$ $\mathrm{HE}_{11}, \mathrm{TE}_{01}, \mathrm{TM}_{01}$, and $\mathrm{HE}_{21}$. For the calculations of this figure, we choose the quantization axis $x_{3}=z$. We observe that $\left|\Omega_{F M F^{\prime} M^{\prime}}\right|$ reduces almost exponentially with increasing $r$. The steep slope in the radial dependence of $\left|\Omega_{F M F^{\prime} M^{\prime}}\right|$ is a manifestation of the evanescent-wave behavior of the guided field outside the fiber. The scaling is determined by the modified Bessel function $K_{l}(q r)$, where $l$ is the azimuthal mode order and $q$ is the inverse of the evanescent-wave penetration length (see Appendix C). The parameter $q$ depends on the mode type. When $r$ is large enough, the function $K_{l}(q r)$ can be approximated as $e^{-q r} \sqrt{\pi / 2 q r}$. It is clear from Fig. 2 that $\left|\Omega_{F M F^{\prime} M^{\prime}}\right|$ depends on not only the magnetic quantum numbers of the atomic transition but also the type of the guided mode. The dotted blue curve in Fig. 2(b), which stands for the case of the upper sublevel $M^{\prime}=2$ and the TE mode, is zero. This means that the TE mode does not interact with the quadrupole transition between the sublevels $|F=2, M=2\rangle$ and $\left|F^{\prime}=4, M^{\prime}=2\right\rangle$ for the quantization axis $x_{3}=z$. The vanishing of this interaction is a consequence of the properties of the TE mode and the quadrupole operator $Q_{i j}$ for the transition $|F=2, M=2\rangle \rightarrow\left|F^{\prime}=4, M^{\prime}=2\right\rangle$ with $x_{3}=z$.

The Rabi frequency $\Omega_{F M F^{\prime} M^{\prime}}$ for the transition between the sublevels $|F M\rangle$ and $\left|F^{\prime} M^{\prime}\right\rangle$ depends on the relative orientation of the quantization axis $x_{3}$ with respect to the fiber axis $z$.
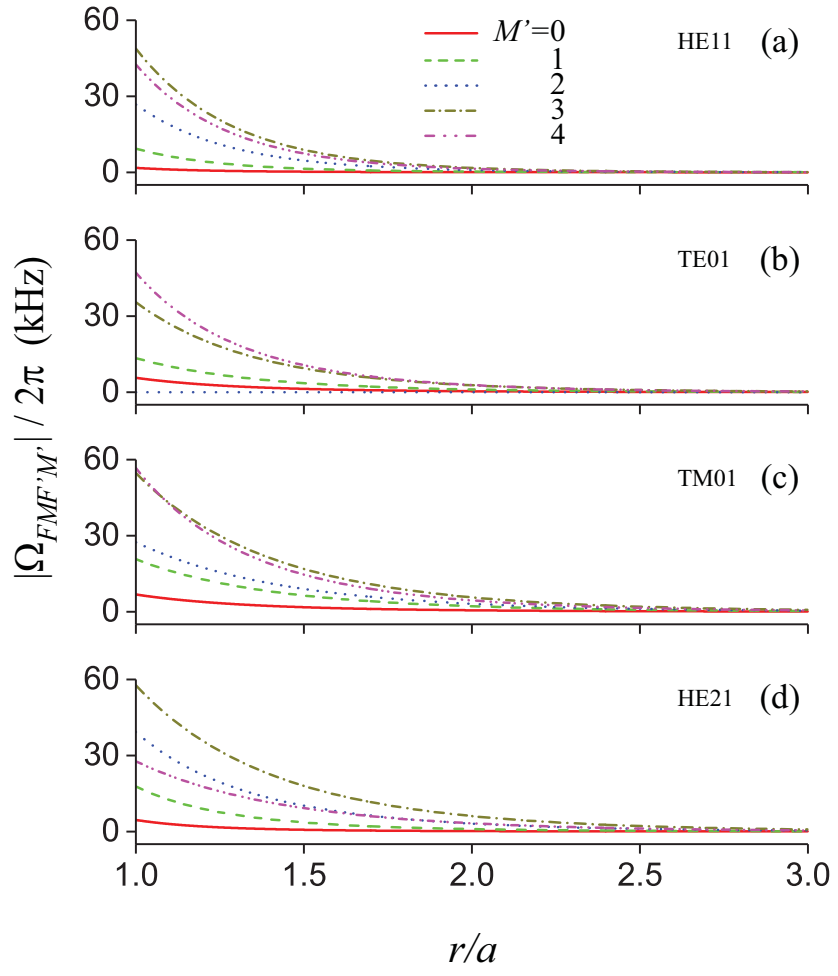

FIG. 2. Absolute value of the Rabi frequency $\Omega_{F M F^{\prime} M^{\prime}}$ for the quadrupole transition between the sublevel $M=2$ of the level $5 S_{1 / 2} F=2$ and a sublevel $M^{\prime}$ of the level $4 D_{5 / 2} F^{\prime}=4$ as a function of the radial distance $r$ for different magnetic quantum numbers $M^{\prime}=0,1,2,3,4$ and different guided mode types $N=$ $\mathrm{HE}_{11}, \mathrm{TE}_{01}, \mathrm{TM}_{01}$, and $\mathrm{HE}_{21}$. The fiber radius is $a=280 \mathrm{~nm}$. The wavelength of the atomic transition is $\lambda_{0}=516.5 \mathrm{~nm}$. The refractive indices of the fiber and the vacuum cladding are $n_{1}=1.4615$ and $n_{2}=1$, respectively. The power of the guided light field is $2.5 \mathrm{nW}$. The field propagates in the $+z$ direction. The hybrid modes are counterclockwise quasicircularly polarized. The quantization axis is $x_{3}=z$. The azimuthal angle for the position of the atom in the fiber cross-section $x y$ plane is arbitrary.

In order to illustrate this dependence, we plot in Fig. 3 the radial dependencies of the absolute value of the Rabi frequency $\Omega_{F M F^{\prime} M^{\prime}}$ for the quadrupole transition between the sublevels $|F=2, M=2\rangle$ and $\left|F^{\prime}=4, M^{\prime}=4\right\rangle$ for different choices of the quantization axis, namely, $x_{3}=z, x$, and $y$. We observe that $\Omega_{F M F^{\prime} M^{\prime}}$ strongly depends on the orientation of $x_{3}$. In the cases of the $\mathrm{HE}_{11}, \mathrm{TM}_{01}$, and $\mathrm{HE}_{21}$ modes, the absolute value $\left|\Omega_{F M F^{\prime} M^{\prime}}\right|$ for $x_{3}=y$ [see the dotted blue curves in Figs. 3(a), $3(\mathrm{c})$, and 3(d)] is larger than for $x_{3}=z$ and $x_{3}=x$ [see the solid black and dashed red curves in Figs. 3(a), 3(c), and 3(d)]. However, in the case of the $\mathrm{TE}_{01}$ mode, we have $\left|\Omega_{F M F^{\prime} M^{\prime}}\right|=0$ for $x_{3}=y$ [see the dotted blue curve in Fig. 3(b)]. The vanishing of this interaction is a consequence of the properties of the TE mode and the quadrupole operator $Q_{i j}$ for the transition $|F=2, M=2\rangle \rightarrow\left|F^{\prime}=4, M^{\prime}=4\right\rangle$ with $x_{3}=y$.

We plot in Figs. 4 and 5 the radial dependencies of $\left|\Omega_{F M F^{\prime} M^{\prime}}\right|$ for the opposite phase circulation directions $p= \pm 1$ and the opposite propagation directions $f= \pm 1$. The dependence of $\left|\Omega_{F M F^{\prime} M^{\prime}}\right|$ on $f$ is related to the spin-orbit coupling of light [41-47]. It has been shown that, due to the spin-orbit coupling of light, spontaneous emission and 


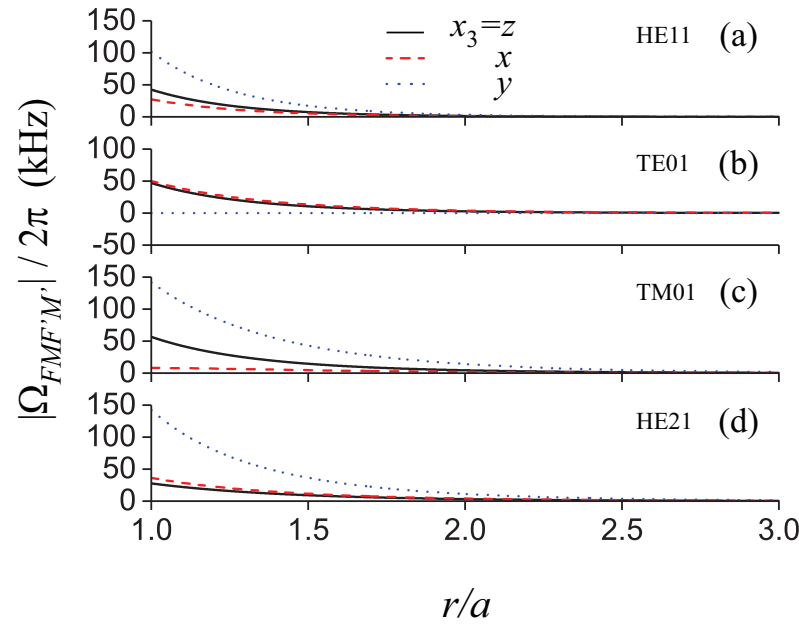

FIG. 3. Radial dependencies of the absolute value of the Rabi frequency $\Omega_{F M F^{\prime} M^{\prime}}$ for the quadrupole transition between the sublevels $|F=2, M=2\rangle$ and $\left|F^{\prime}=4, M^{\prime}=4\right\rangle$ for different choices of the quantization axis $x_{3}$ and different guided modes. The atom is positioned on the positive side of the $x$ axis $(\varphi=0)$ and the hybrid modes are counterclockwise quasicircularly polarized. Other parameters are as for Fig. 2.

scattering from an atom with a circular dipole near a nanofiber can be asymmetric with respect to the opposite propagation directions along the fiber axis [48-54]. We note that we have $\left|\Omega_{F M F^{\prime} M^{\prime}}\right|=0$ for both directions $f= \pm 1$ in Fig. 5(c), where $M=2, M^{\prime}=4$, and $N=\mathrm{TE}_{01}$, and in Fig. 5(f), where $M=2, M^{\prime}=3$, and $N=\mathrm{TM}_{01}$. Our additional analysis, which is not shown here, confirms the vanishing of these quadrupole transitions.

Next, we calculate the rms Rabi frequency $\bar{\Omega}_{F F^{\prime}}$ and the oscillator strength $f_{F F^{\prime}}$ as functions of the position $r$ of the atom and the radius $a$ of the fiber. As already pointed out in

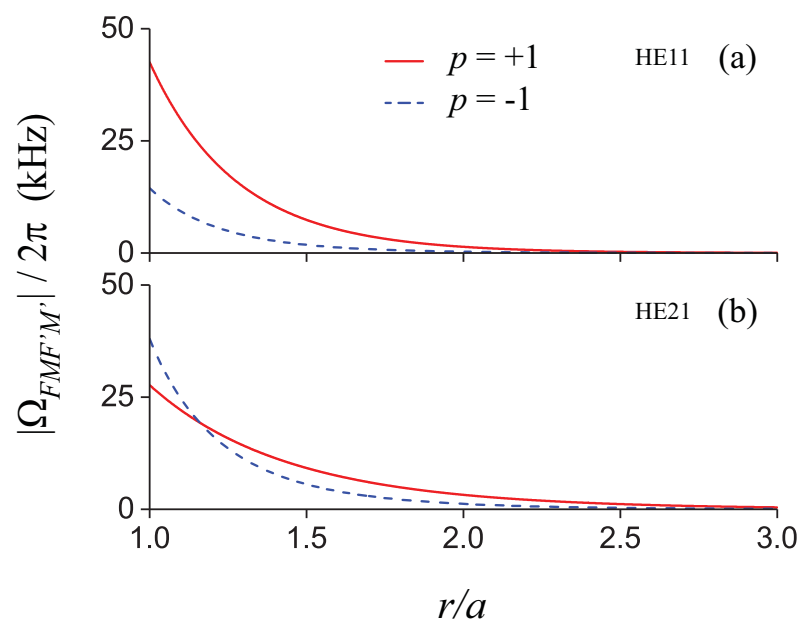

FIG. 4. Radial dependencies of the absolute value of the Rabi frequency $\Omega_{F M F^{\prime} M^{\prime}}$ for the opposite phase circulation directions $p=$ \pm 1 of the circularly polarized hybrid modes $\mathrm{HE}_{11}$ and $\mathrm{HE}_{21}$. The lower and upper levels of the transition are $|F=2, M=2\rangle$ and $\mid F^{\prime}=$ $\left.4, M^{\prime}=4\right\rangle$ and the quantization axis is $x_{3}=z$. Other parameters are as for Fig. 2.

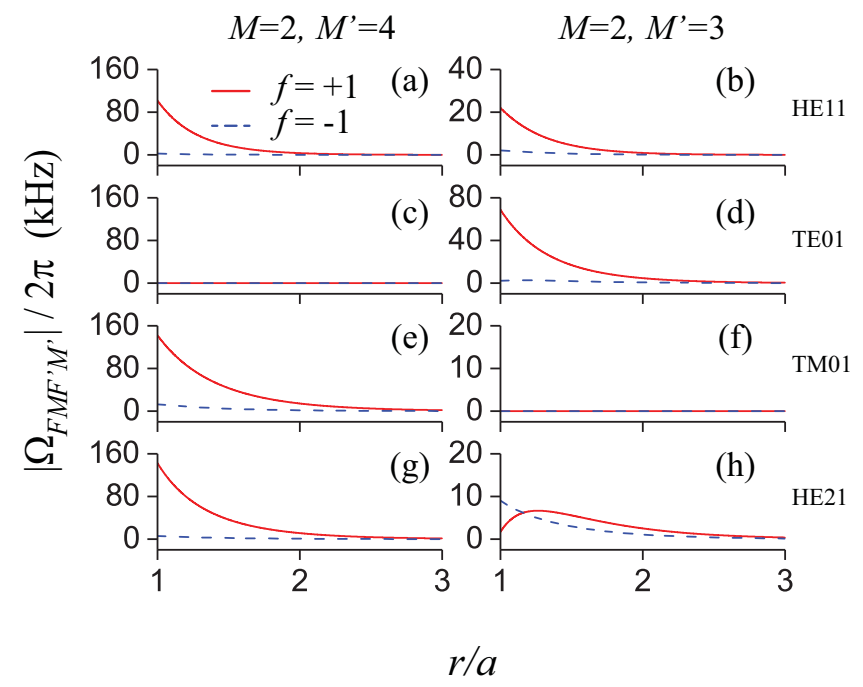

FIG. 5. Radial dependencies of the absolute value of the Rabi frequency $\Omega_{F M F^{\prime} M^{\prime}}$ for the opposite propagation directions $f= \pm 1$ of different guided modes. The lower and upper levels of the transition are $|F=2, M=2\rangle$ and $\left|F^{\prime}=4, M^{\prime}=4\right\rangle$ (left column) and $\mid F=$ $2, M=2\rangle$ and $\left|F^{\prime}=4, M^{\prime}=3\right\rangle$ (right column). The quantization axis is $x_{3}=y$, the atom is positioned on the positive side of the $x$ axis, and the hybrid modes are counterclockwise quasicircularly polarized. Other parameters are as for Fig. 2.

Sec. II, due to the summation over transitions with different magnetic quantum numbers, $\bar{\Omega}_{F F^{\prime}}$ and $f_{F F^{\prime}}$ do not depend on the relative orientation of the quantization axis $x_{3}$ with respect to fiber axis $z$. Moreover, the shapes of the dependencies of $\bar{\Omega}_{F F^{\prime}}$ and $f_{F F^{\prime}}$ on $r$ and $a$ do not depend on $F$ and $F^{\prime}$.

We plot in Figs. 6 and 7 the radial dependencies of the rms Rabi frequency $\bar{\Omega}_{F F^{\prime}}$ and the oscillator strength $f_{F F^{\prime}}$ of the atom. These figures show that $\bar{\Omega}_{F F^{\prime}}$ and $f_{F F^{\prime}}$ achieve their largest values at $r / a=1$. We observe that $\bar{\Omega}_{F F^{\prime}}$ reduces quickly but $f_{F F^{\prime}}$ decreases slowly with increasing $r$. The reason for the sharp contrast between the dependencies of these quantities on $r$ is that, unlike the Rabi frequency, the

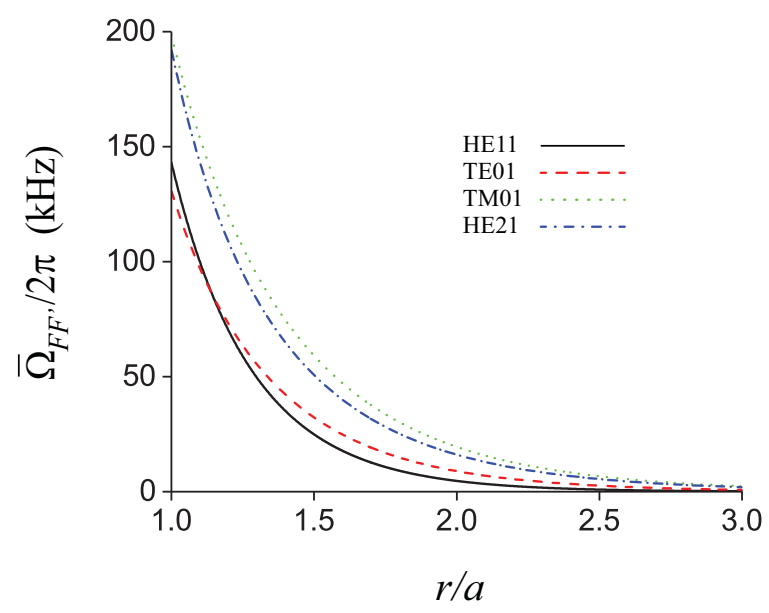

FIG. 6. Radial dependencies of the rms Rabi frequency $\bar{\Omega}_{F F^{\prime}}$ for different guided modes. The hfs levels are $F=2$ and $F^{\prime}=4$. The hybrid modes are quasicircularly polarized and the quantization axis is arbitrary. Other parameters are as for Fig. 2. 


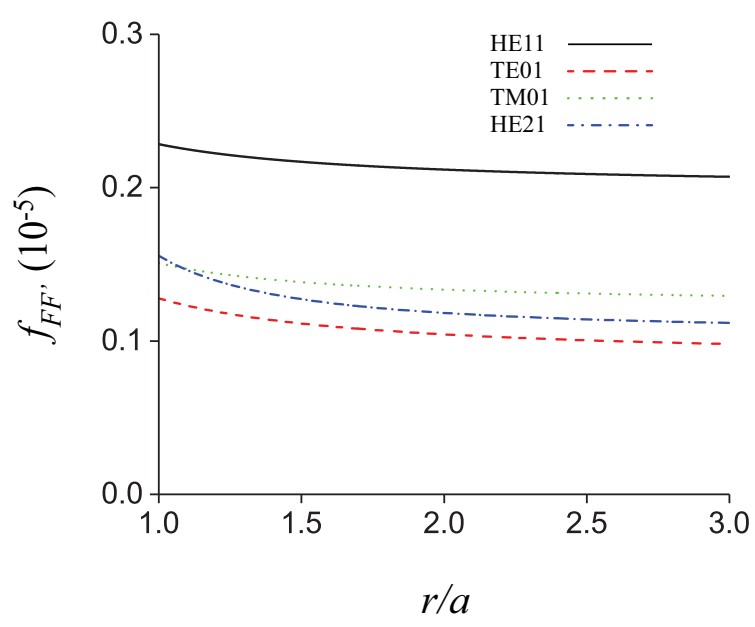

FIG. 7. Radial dependencies of the oscillator strength $f_{F F^{\prime}}$ for different guided modes. Parameters used are as for Fig. 6.

oscillator strength $f_{F F^{\prime}}$ does not depend on the magnitude of the field amplitude. Indeed, $f_{F F^{\prime}}$ is, as already mentioned in the previous section, proportional to the square of the ratio between the field gradient and the field amplitude. In the limit of large radial distances, both the gradient and the amplitude of the field in a guided mode tend to zero but the ratio between them tends to a nonzero value.

Figures 6 and 7 show that the rms Rabi frequency $\bar{\Omega}_{F F^{\prime}}$ and the oscillator strength $f_{F F^{\prime}}$ depend on the mode type. Comparison between the curves for different modes shows that, for the parameters of the figures, the oscillator strength $f_{F F^{\prime}}$ for the fundamental mode $\mathrm{HE}_{11}$ (see the solid black curve in Fig. 7) is the largest, while the corresponding rms Rabi frequency $\bar{\Omega}_{F F^{\prime}}$ (see the solid black curve in Fig. 6) is the smallest or the second smallest. The contrast between these relations is due to the fact that the rms Rabi frequency $\bar{\Omega}_{F F^{\prime}}$ is proportional to the product of the oscillator strength $f_{F F^{\prime}}$ and the electric field intensity $|\mathcal{E}|^{2}$ [see Eq. (9)]. Outside the fiber, for a given power, the magnitude of the intensity of the field in the fundamental mode is smaller than that in other modes [34].

We show in Figs. 8 and 9 the rms Rabi frequency $\bar{\Omega}_{F F^{\prime}}$ and the oscillator strength $f_{F F^{\prime}}$ as functions of the fiber radius $a$. We observe from Fig. 8 that the rms Rabi frequency $\bar{\Omega}_{F F^{\prime}}$ first increases and then decreases with increasing $a$. It is clear from this figure that $\bar{\Omega}_{F F^{\prime}}$ for different guided modes have different maxima at different values of $a$. We observe from Fig. 9 that, for the fundamental mode $\mathrm{HE}_{11}$, the oscillator strength $f_{F F^{\prime}}$ has a local minimum at $a \simeq 107 \mathrm{~nm}$. Meanwhile, for the higherorder modes, $f_{F F^{\prime}}$ increases with increasing $a$. In the region $a<498.2 \mathrm{~nm}, f_{F F^{\prime}}$ for the $\mathrm{HE}_{11}$ mode is larger than that for higher-order modes. When $a$ is in the region from 498.2 to $1000 \mathrm{~nm}, f_{F F^{\prime}}$ for the $\mathrm{TM}_{01}$ mode is larger than that for other modes.

The increase of $f_{F F^{\prime}}$ for the $\mathrm{HE}_{11}$ and higher-order modes with increasing $a$ in the region of large $a$ is a consequence of the fact that expression (11) for $f_{F F^{\prime}}$ contains the terms that are proportional to the gradients $\partial \mathcal{E}_{x, y, z} / \partial z$ of the field amplitudes $\mathcal{E}_{x, y, z}$ in the direction of the fiber axis $z$. These gradients are proportional to the propagation constant $\beta$, which increases with increasing fiber radius $a[34,40]$. The decrease

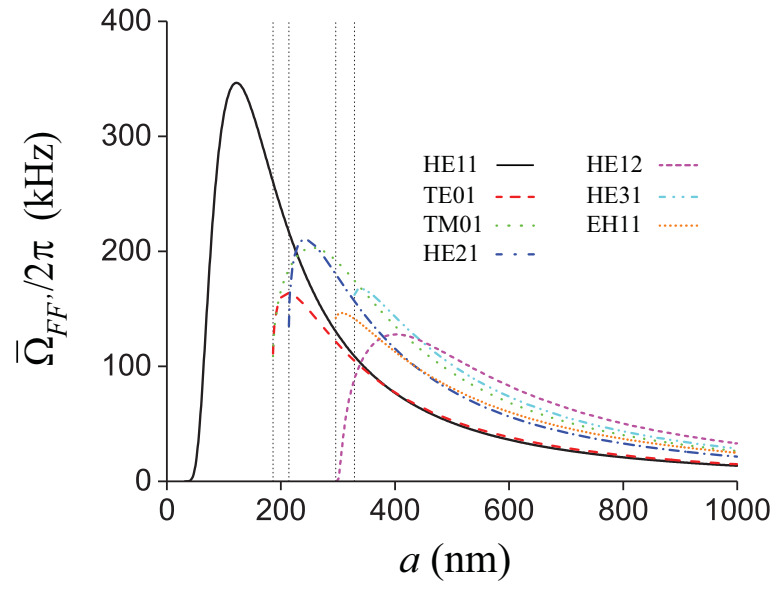

FIG. 8. The rms Rabi frequency $\bar{\Omega}_{F F^{\prime}}$ as a function of the fiber radius $a$ for different guided modes. The atom is positioned on the fiber surface. The hybrid modes are quasicircularly polarized and the quantization axis is arbitrary. Other parameters are as for Fig. 2. The vertical dotted lines indicate the positions of the cutoffs for higher-order modes.

of $f_{F F^{\prime}}$ with increasing $a$ in the region of small $a$ for the $\mathrm{HE}_{11}$ mode (see the solid black curve in Fig. 9) is a result of the changes in the structure of the field. The initial decrease and the subsequent increase lead to the occurrence of a minimum in the dependence of $f_{F F^{\prime}}$ on $a$ in the case of the $\mathrm{HE}_{11}$ mode (see the solid black curve in Fig. 9).

We now calculate the oscillator-strength enhancement factor $\eta_{\text {osc }}$, which is the ratio between the oscillator strength $f_{F F^{\prime}}$ for a fiber guided field and the oscillator strength $f_{F F^{\prime}}^{(0)}$ for a free-space plane-wave field. As already pointed out in Sec. II, the magnitude of the enhancement factor $\eta_{\text {osc }}$ does not depend on the quantum numbers $F$ and $F^{\prime}$ and any other characteristics of the atomic states except for the atomic transition frequency $\omega_{0}$. Like $f_{F F^{\prime}}$, the enhancement factor $\eta_{\text {osc }}$ does not depend on the magnitude $\mathcal{E}$ of the field amplitude.

We plot in Fig. 10 the radial dependencies of the oscillatorstrength enhancement factor $\eta_{\text {osc }}$ for different guided modes.

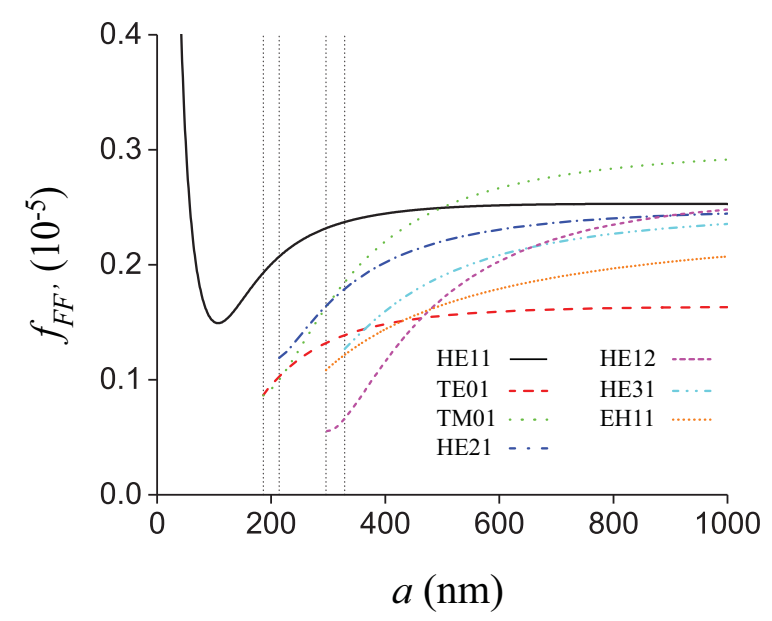

FIG. 9. Oscillator strength $f_{F F^{\prime}}$ as a function of the fiber radius $a$ for different guided modes. Parameters used are as for Fig. 8. 


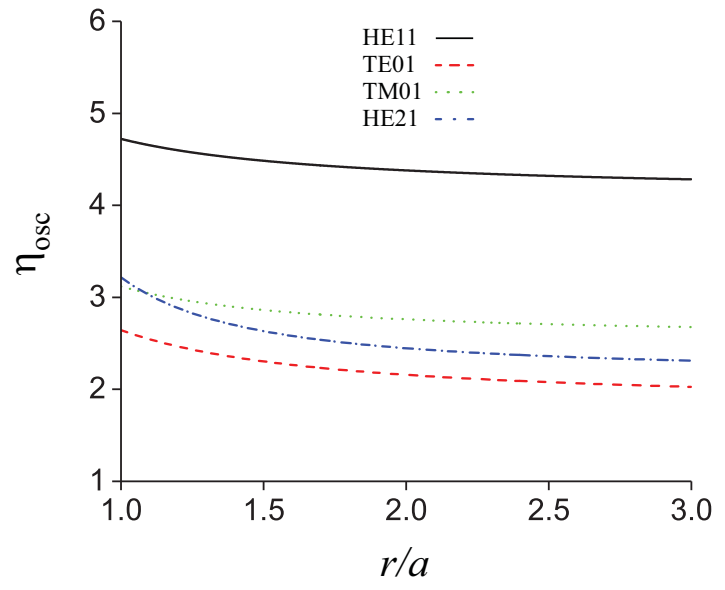

FIG. 10. Radial dependencies of the oscillator-strength enhancement factor $\eta_{\text {osc }}$ for different guided modes. The hybrid modes are quasicircularly polarized and the quantization axis is arbitrary. Other parameters are as for Fig. 2.

It is clear from the figure that $\eta_{\mathrm{osc}}$ achieves its largest values at $r / a=1$. We see that $\eta_{\text {osc }}$ reduces slowly with increasing radial distance $r$. This result means that, despite the evanescent wave behavior, the enhancement factor $\eta_{\text {osc }}$ can be significant even when the atom is far away from the fiber. The reason is that the oscillator strength $f_{F F^{\prime}}$ and consequently the enhancement factor $\eta_{\text {osc }}$ are determined by not the field amplitude but the ratio between the field gradient and the field amplitude. We emphasize that guided modes are different from freespace plane waves even in the limit of large radial distances. Therefore, the enhancement factor $\eta_{\mathrm{osc}}$ can be different from 1 even in this limit [see Eq. (24)].

We show in Fig. 11 the oscillator-strength enhancement factor $\eta_{\text {osc }}$ as a function of the fiber radius $a$ for different guided modes. Similar to the oscillator strength $f_{F F^{\prime}}$, the enhancement factor $\eta_{\mathrm{osc}}$ for the fundamental mode $\mathrm{HE}_{11}$ has a local minimum

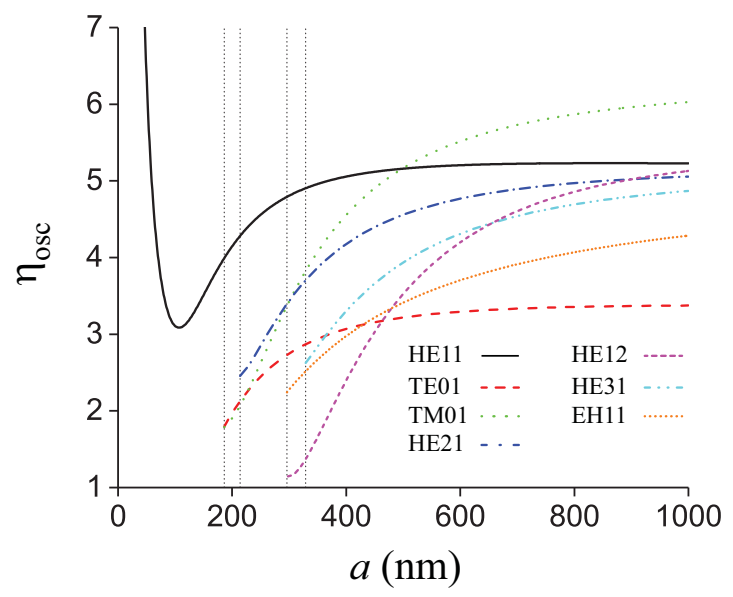

FIG. 11. Oscillator-strength enhancement factor $\eta_{\text {osc }}$ as a function of the fiber radius $a$ for different guided modes. The atom is positioned on the fiber surface. The hybrid modes are quasicircularly polarized and the quantization axis is arbitrary. Other parameters are as for Fig. 2. The vertical dotted lines indicate the positions of the cutoffs for higher-order modes.

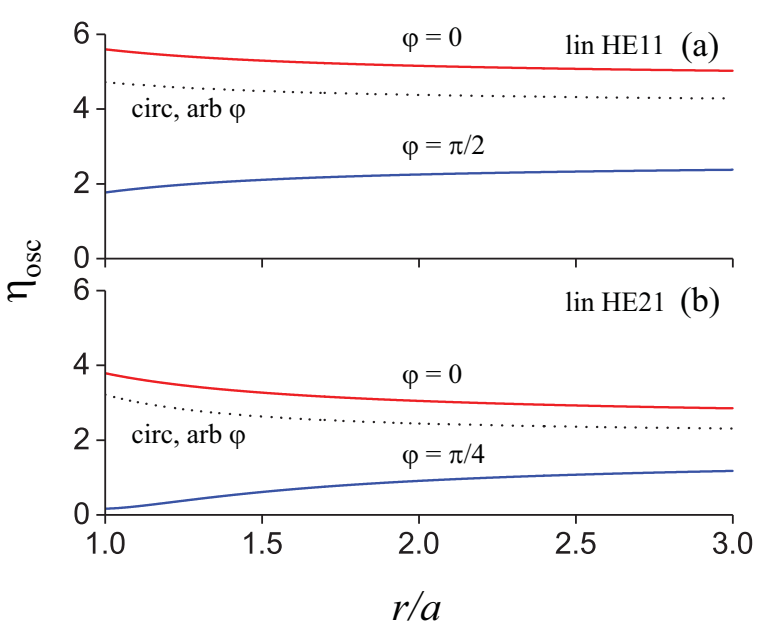

FIG. 12. Oscillator-strength enhancement factors $\eta_{\text {osc }}$ for the quasilinearly polarized $\mathrm{HE}_{11}$ and $\mathrm{HE}_{21}$ modes as functions of the radial distance $r$ at different azimuthal angles $\varphi$. The orientation angle of the quasilinear polarization axis is $\varphi_{\mathrm{pol}}=0$ and the quantization axis is arbitrary. Other parameters are as for Fig. 2. For comparison, the results for the corresponding quasicircularly polarized hybrid modes are shown by the dotted black curves.

at the fiber radius $a \simeq 107 \mathrm{~nm}$, and is larger than that for higherorder modes in the region $a<498.2 \mathrm{~nm}$. Meanwhile, the enhancement factor $\eta_{\text {osc }}$ for higher-order modes monotonically increases with increasing $a$. When $a$ is in the region from 498.2 to $1000 \mathrm{~nm}$, the factor $\eta_{\mathrm{osc}}$ for the $\mathrm{TM}_{01}$ mode is larger than that for other modes.

Due to the summation over transitions with different magnetic quantum numbers and the cylindrical symmetry of the

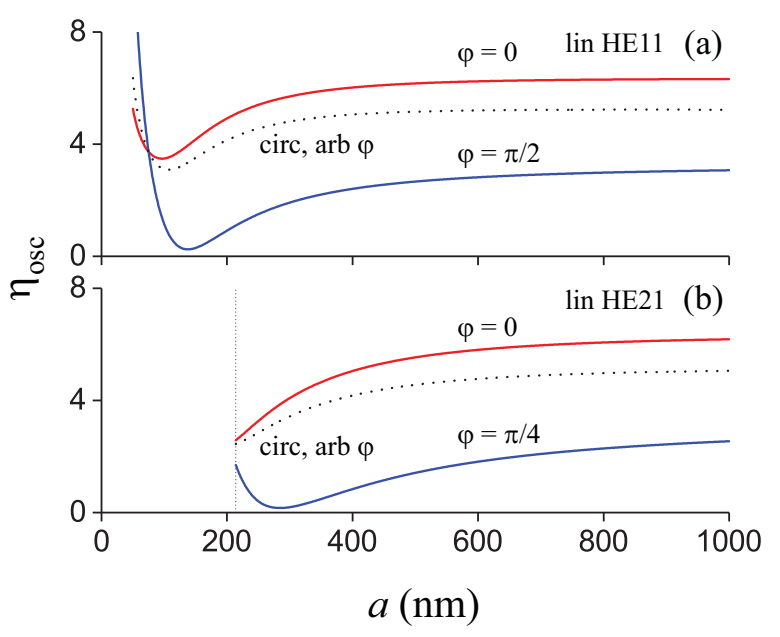

FIG. 13. Oscillator-strength enhancement factors $\eta_{\text {osc }}$ for the quasilinearly polarized $\mathrm{HE}_{11}$ and $\mathrm{HE}_{21}$ modes as functions of the fiber radius $a$. The atom is positioned on the fiber surface at different azimuthal angles $\varphi$. The orientation angle of the quasilinear polarization axis is $\varphi_{\mathrm{pol}}=0$ and the quantization axis is arbitrary. Other parameters are as for Fig. 2. The vertical dotted line indicates the position of the cutoff for the $\mathrm{HE}_{21}$ mode. For comparison, the results for the corresponding quasicircularly polarized hybrid modes are shown by the dotted black curves. 


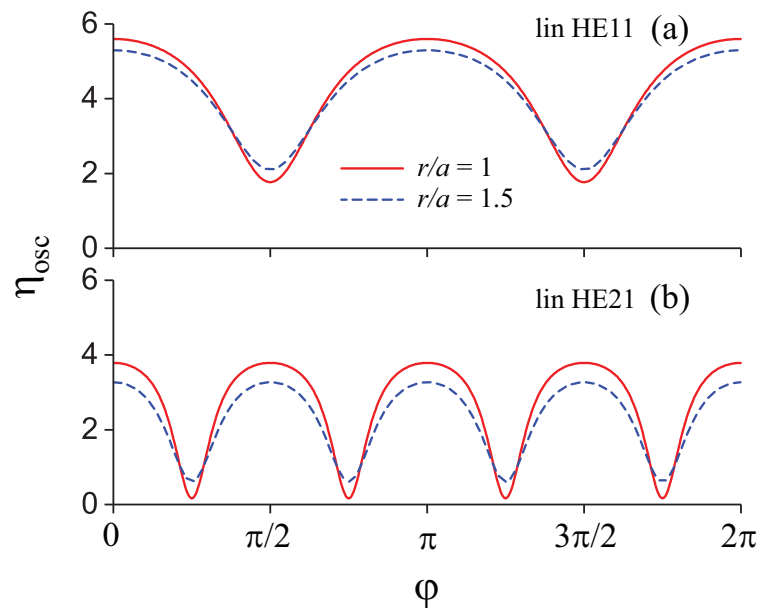

FIG. 14. Oscillator-strength enhancement factors $\eta_{\text {osc }}$ for the quasilinearly polarized $\mathrm{HE}_{11}$ and $\mathrm{HE}_{21}$ modes as functions of the azimuthal angle $\varphi$ for the position of the atom in the fiber cross-section plane. The orientation angle of the quasilinear polarization axis is $\varphi_{\mathrm{pol}}=0$ and the quantization axis is arbitrary. Other parameters are as for Fig. 2.

field in a quasicircularly polarized hybrid mode, the oscillator strength $f_{F F^{\prime}}$ and the enhancement factor $\eta_{\text {osc }}$ for such a mode do not depend on the azimuthal position $\varphi$ of the atom in the fiber transverse plane. For the field in a quasilinearly polarized hybrid mode, since the cylindrical symmetry is broken, $f_{F F^{\prime}}$ and $\eta_{\text {osc }}$ vary with varying $\varphi$. We plot in Figs. 12 and 13 the

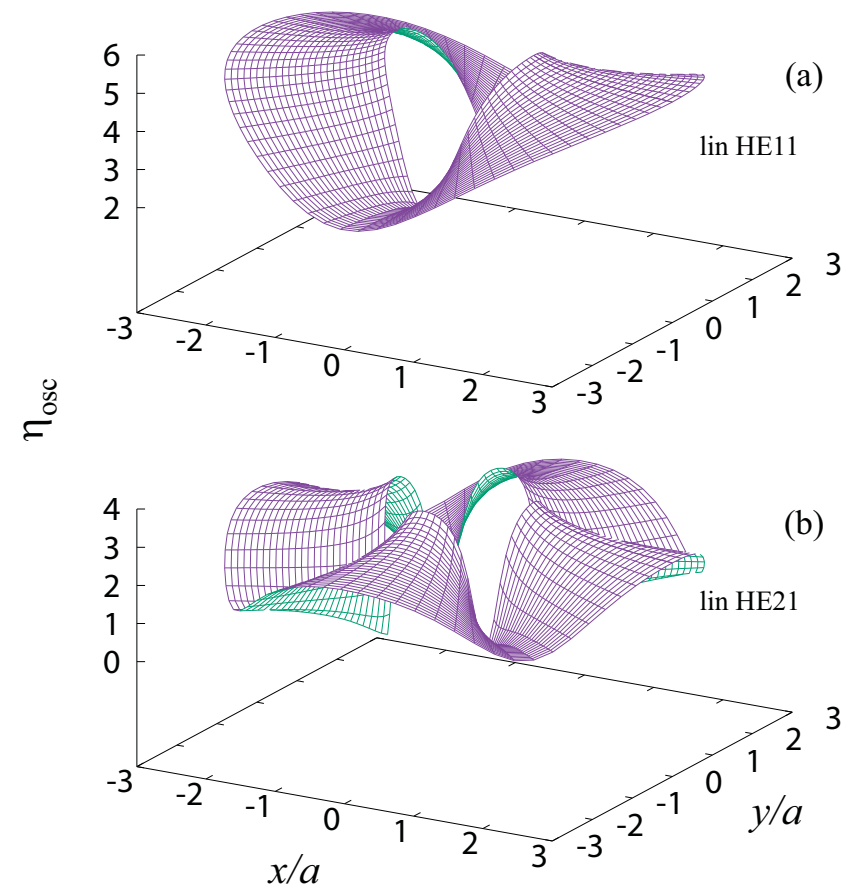

FIG. 15. Oscillator-strength enhancement factors $\eta_{\text {osc }}$ for the quasilinearly polarized $\mathrm{HE}_{11}$ and $\mathrm{HE}_{21}$ modes as functions of the position of the atom in the fiber cross-section plane. The orientation angle of the quasilinear polarization axis is $\varphi_{\text {pol }}=0$ and the quantization axis is arbitrary. Other parameters are as for Fig. 2. dependencies of $\eta_{\text {osc }}$ for the quasilinearly polarized $\mathrm{HE}_{11}$ and $\mathrm{HE}_{21}$ modes on the radial distance $r$ and the fiber radius $a$ for different azimuthal angles $\varphi$. We observe from the figures that, depending on $\varphi$, the factor $\eta_{\text {osc }}$ for a quasilinearly polarized hybrid mode may decrease or increase with increasing distance $r$, may be larger or smaller than that for the corresponding quasicircularly polarized hybrid mode, and may have a minimum in the dependence on the fiber radius $a$. Figure 12 shows that $\eta_{\text {osc }}$ varies slowly in the radial direction. Comparison between the curves for different azimuthal angles in Figs. 12 and 13 indicates that $\eta_{\text {osc }}$ for quasilinearly polarized modes varies significantly in the azimuthal direction.

In order to get a better view of the spatial profiles of the enhancement factor $\eta_{\text {osc }}$ for quasilinearly polarized hybrid modes, we plot in Figs. 14 and 15 this factor as a function of the azimuthal angle $\varphi$ and as a function of the Cartesian coordinates $x$ and $y$ of the position of the atom in the fiber cross-section plane. The figures show that $\eta_{\text {osc }}$ for quasilinearly polarized hybrid modes varies significantly in the azimuthal direction but slightly in the radial direction, and is relatively large or small along the major or minor symmetry axes of the modes, respectively.

\section{CONCLUSION AND DISCUSSION}

In this work, we have studied the electric quadrupole interaction of an alkali-metal atom with guided light in the fundamental and higher-order modes of a vacuum-clad ultrathin optical fiber. We have calculated the quadrupole Rabi frequency, the quadrupole oscillator strength, and their enhancement factors. In the example of a ${ }^{87} \mathrm{Rb}$ atom, we have studied the dependencies of the Rabi frequency on the quantum numbers of the transition, the mode type, the phase circulation direction, the propagation direction, the orientation of the quantization axis, the position of the atom, and the fiber radius. We have found that the rms quadrupole Rabi frequency and the quadrupole oscillator strength are enhanced by the effect of the fiber on the gradient of the field amplitude. With increasing radial distance, the rms Rabi frequency reduces quickly but the oscillator strength varies slowly. The enhancement factors of the rms Rabi frequency and the oscillator strength do not depend on any characteristics of the internal atomic states except for the atomic transition frequency. These factors are determined by the ratios between the gradients and the amplitude of the field operating at the atomic transition frequency. Like the oscillator strength, its enhancement factor $\eta_{\text {osc }}$ varies slowly with increasing distance from the atom to the fiber surface. Due to this fact, the factor $\eta_{\text {osc }}$ can be significant even when the atom is far away from the fiber. We have found that, in the case where the atom is positioned on the fiber surface, the oscillator strength for the quasicircularly polarized fundamental mode $\mathrm{HE}_{11}$ has a local minimum at the fiber radius $a \simeq 107 \mathrm{~nm}$. Meanwhile, for quasicircularly polarized higher-order hybrid modes, transverse electric modes, and transverse magnetic modes, the oscillator strength monotonically increases with increasing $a$. In the region $a<498.2 \mathrm{~nm}$, the oscillator strength for the quasicircularly polarized $\mathrm{HE}_{11}$ mode is larger than that for quasicircularly polarized higher-order hybrid modes, transverse electric modes, and transverse magnetic modes. We have shown that, depending on the azimuthal position of the atom, 
the enhancement factor $\eta_{\text {osc }}$ for a quasilinearly polarized hybrid mode may decrease or increase with increasing distance, and may be larger or smaller than that for the corresponding quasicircularly polarized hybrid mode. We have found that the factor $\eta_{\text {osc }}$ for quasilinearly polarized hybrid modes varies significantly in the azimuthal direction, and is relatively large or small along the major or minor symmetry axes of the modes, respectively.

Our results may find application in future research on probing electric quadrupole transitions of atoms, molecules, and particles using the fundamental and higher-order modes of ultrathin optical fibers. Direct access to electric quadrupole transitions might be beneficial for fiber-based optical clocks [55]. A photon in a higher-order hybrid mode may have significant orbital angular momentum in addition to spin angular momentum. Therefore, our results on the enhanced electric quadrupole interaction of an atom with guided light might lead to an efficient way for transferring more than one quantum of angular momentum per photon to the internal degrees of freedom of the atom [8,14]. Furthermore, the particular atomic transition addressed in this article allows one to prepare a rubidium atom in the excited state $4 D_{5 / 2}$. The only dipole-allowed decay of this state to the ground state is via the intermediate level $5 P_{3 / 2}$, by cascaded emission of two photons at 1530 and $780 \mathrm{~nm}$. The emitted photons are correlated and can be entangled [56,57]. This opens up the possibility to develop a fiber-based source of entangled photon pairs at wavelengths relevant to telecom and atomic references.

\section{ACKNOWLEDGMENT}

We acknowledge support for this work from the Okinawa Institute of Science and Technology Graduate University.

\section{APPENDIX A: MATRIX ELEMENTS OF THE QUADRUPOLE TENSOR OPERATORS}

We introduce the notations

$$
\begin{aligned}
& x_{-1}^{(1)}=\frac{x_{1}-i x_{2}}{\sqrt{2}}, \\
& x_{0}^{(1)}=x_{3}, \\
& x_{1}^{(1)}=-\frac{x_{1}+i x_{2}}{\sqrt{2}},
\end{aligned}
$$

for the spherical tensor components of the position vector $\mathbf{x}=$ $\left(x_{1}, x_{2}, x_{3}\right)$. In terms of these components, we have

$$
\begin{aligned}
& x_{1}=\frac{x_{-1}^{(1)}-x_{1}^{(1)}}{\sqrt{2}}, \\
& x_{2}=i \frac{x_{-1}^{(1)}+x_{1}^{(1)}}{\sqrt{2}}, \\
& x_{3}=x_{0}^{(1)} .
\end{aligned}
$$

We can write

$$
x_{i}=\sum_{q} u_{i}^{(q)} x_{q}^{(1)}
$$

where $u_{i}^{(q)}$ with $i=1,2,3$ are the components of the spherical basis vectors $\mathbf{u}^{(q)}$ in the Cartesian coordinate system $\left\{x_{1}, x_{2}, x_{3}\right\}$. The expressions for the vectors $\mathbf{u}^{(q)}$ are

$$
\begin{aligned}
\mathbf{u}^{(-1)} & =\frac{1}{\sqrt{2}}(1, i, 0), \\
\mathbf{u}^{(0)} & =(0,0,1), \\
\mathbf{u}^{(1)} & =-\frac{1}{\sqrt{2}}(1,-i, 0) .
\end{aligned}
$$

We note that $\mathbf{u}^{(q) *}=(-1)^{q} \mathbf{u}^{(-q)}, \quad \mathbf{u}^{(q)} \cdot \mathbf{u}^{\left(q^{\prime}\right) *}=\delta_{q q^{\prime}}$, and $\sum_{q} u_{i}^{(q)} u_{j}^{(q) *}=\delta_{i j}$.

It follows from Eq. (A3) that

$$
x_{i} x_{j}=\sum_{M_{1} M_{2}} u_{i}^{\left(M_{1}\right)} u_{j}^{\left(M_{2}\right)} x_{M_{1}}^{(1)} x_{M_{2}}^{(1)}
$$

In order to calculate the direct product $x_{M_{1}}^{(1)} x_{M_{2}}^{(1)}$, we use the formula [58]

$$
x_{M_{1}}^{(1)} x_{M_{2}}^{(1)}=\sum_{K q} T_{q}^{(K)}(-1)^{q} \sqrt{2 K+1}\left(\begin{array}{ccc}
1 & 1 & K \\
M_{1} & M_{2} & -q
\end{array}\right) \text {, }
$$

where $T_{q}^{(K)}$ with $q=-K, \ldots, K$ are the tensor elements of the irreducible tensor products $T^{(K)}=\left[x^{(1)} \otimes x^{(1)}\right]^{(K)}$ of rank $K=0,1,2$. The expression for $T_{q}^{(K)}$ is

$$
T_{q}^{(K)}=(-1)^{q} \sqrt{2 K+1} \sum_{q_{1} q_{2}}\left(\begin{array}{ccc}
1 & 1 & K \\
q_{1} & q_{2} & -q
\end{array}\right) x_{q_{1}}^{(1)} x_{q_{2}}^{(1)} .
$$

We can show that

$$
T_{0}^{(0)}=-\frac{x_{1}^{2}+x_{2}^{2}+x_{3}^{2}}{\sqrt{3}}, \quad T_{q}^{(1)}=0,
$$

and

$$
\begin{aligned}
& T_{0}^{(2)}=\frac{2 x_{3}^{2}-x_{1}^{2}-x_{2}^{2}}{\sqrt{6}}, \\
& T_{1}^{(2)}=-x_{3}\left(x_{1}+i x_{2}\right), \\
& T_{-1}^{(2)}=x_{3}\left(x_{1}-i x_{2}\right), \\
& T_{2}^{(2)}=\frac{1}{2}\left(x_{1}+i x_{2}\right)^{2}, \\
& T_{-2}^{(2)}=\frac{1}{2}\left(x_{1}-i x_{2}\right)^{2} .
\end{aligned}
$$

Note that $T_{0}^{(0)}=-R^{2} / \sqrt{3}$ and $T_{q}^{(2)}=2(2 \pi / 15)^{1 / 2} R^{2} Y_{2 q}$ $(\vartheta, \phi)$, where $Y_{l q}(\vartheta, \phi)$ are spherical harmonics with $\vartheta$ and $\phi$ being spherical angles.

We insert Eq. (A6) into Eq. (A5) and use Eq. (1). Then, we obtain

$$
Q_{i j} \equiv e\left(3 x_{i} x_{j}-R^{2} \delta_{i j}\right)=3 e \sum_{q} u_{i j}^{(q)} T_{q}^{(2)},
$$


where

$$
u_{i j}^{(q)}=(-1)^{q} \sqrt{5} \sum_{M_{1} M_{2}} u_{i}^{\left(M_{1}\right)} u_{j}^{\left(M_{2}\right)}\left(\begin{array}{ccc}
1 & 1 & 2 \\
M_{1} & M_{2} & -q
\end{array}\right) .
$$

The explicit expressions for the tensors $u_{i j}^{(q)}$ are

$$
\begin{aligned}
u_{i j}^{(2)} & =\frac{1}{2}\left(\begin{array}{ccc}
1 & -i & 0 \\
-i & -1 & 0 \\
0 & 0 & 0
\end{array}\right), \\
u_{i j}^{(1)} & =\frac{1}{2}\left(\begin{array}{ccc}
0 & 0 & -1 \\
0 & 0 & i \\
-1 & i & 0
\end{array}\right), \\
u_{i j}^{(0)} & =\frac{1}{\sqrt{6}}\left(\begin{array}{ccc}
-1 & 0 & 0 \\
0 & -1 & 0 \\
0 & 0 & 2
\end{array}\right), \\
u_{i j}^{(-1)} & =\frac{1}{2}\left(\begin{array}{ccc}
0 & 0 & 1 \\
0 & 0 & i \\
1 & i & 0
\end{array}\right), \\
u_{i j}^{(-2)} & =\frac{1}{2}\left(\begin{array}{ccc}
1 & i & 0 \\
i & -1 & 0 \\
0 & 0 & 0
\end{array}\right) .
\end{aligned}
$$

Note that $u_{i j}^{(q)}=u_{j i}^{(q)}, u_{i j}^{(q) *}=(-1)^{q} u_{i j}^{(-q)}, \sum_{i j} u_{i j}^{(q)} u_{i j}^{\left(q^{\prime}\right) *}=$ $\delta_{q q^{\prime}}$, and $\sum_{i} u_{i i}^{(q)}=0$.

The matrix elements of the tensor $T_{q}^{(2)}$ can be calculated using the Wigner-Eckart theorem [58],

$$
\begin{aligned}
& \left\langle n^{\prime} F^{\prime} M^{\prime}\left|T_{q}^{(2)}\right| n F M\right\rangle \\
& =(-1)^{F^{\prime}-M^{\prime}}\left(\begin{array}{ccc}
F^{\prime} & 2 & F \\
-M^{\prime} & q & M
\end{array}\right)\left\langle n^{\prime} F^{\prime}\left\|T^{(2)}\right\| n F\right\rangle,
\end{aligned}
$$

where the array in the parentheses is a $3 j$ symbol and the invariant factor $\left\langle n^{\prime} F^{\prime}\left\|T^{(2)}\right\| n F\right\rangle$ is a reduced matrix element. The selection rules for $F$ and $F^{\prime}$ are $\left|F^{\prime}-F\right| \leqslant 2 \leqslant F^{\prime}+F$. The selection rules for $M$ and $M^{\prime}$ are $\left|M^{\prime}-M\right| \leqslant 2$ and $M^{\prime}-$ $M=q$. When we use Eqs. (A10) and (A13), we obtain [7]

$$
\begin{aligned}
& \left\langle n^{\prime} F^{\prime} M^{\prime}\left|Q_{i j}\right| n F M\right\rangle \\
& =3 e u_{i j}^{\left(M^{\prime}-M\right)}(-1)^{F^{\prime}-M^{\prime}}\left(\begin{array}{ccc}
F^{\prime} & 2 & F \\
-M^{\prime} & M^{\prime}-M & M
\end{array}\right) \\
& \quad \times\left\langle n^{\prime} F^{\prime}\left\|T^{(2)}\right\| n F\right\rangle .
\end{aligned}
$$

Since the tensor operators $T_{q}^{(2)}$ do not act on the nuclear spin degree of freedom, the dependence of the reduced matrix element $\left\langle n^{\prime} F^{\prime}\left\|T^{(2)}\right\| n F\right\rangle$ on $F$ and $F^{\prime}$ may be factored out as [58]

$$
\begin{aligned}
& \left\langle n^{\prime} F^{\prime}\left\|T^{(2)}\right\| n F\right\rangle \\
& =(-1)^{J^{\prime}+I+F} \sqrt{(2 F+1)\left(2 F^{\prime}+1\right)}\left\{\begin{array}{ccc}
F^{\prime} & 2 & F \\
J & I & J^{\prime}
\end{array}\right\} \\
& \quad \times\left\langle n^{\prime} J^{\prime}\left\|T^{(2)}\right\| n J\right\rangle,
\end{aligned}
$$

where $J$ is the quantum number for the total angular momentum of the electrons, $I$ is the nuclear spin quantum number, and the array in the curly braces is a $6 j$ symbol. The selection rules for $J$ and $J^{\prime}$ are $\left|J^{\prime}-J\right| \leqslant 2 \leqslant J^{\prime}+J$.

Furthermore, since the tensor operators $T_{q}^{(2)}$ do not act on the electron spin degree of freedom, we have [58]

$$
\begin{aligned}
& \left\langle n^{\prime} J^{\prime}\left\|T^{(2)}\right\| n J\right\rangle \\
& =(-1)^{L^{\prime}+S+J} \sqrt{(2 J+1)\left(2 J^{\prime}+1\right)}\left\{\begin{array}{lll}
J^{\prime} & 2 & J \\
L & S & L^{\prime}
\end{array}\right\} \\
& \quad \times\left\langle n^{\prime} L^{\prime}\left\|T^{(2)}\right\| n L\right\rangle,
\end{aligned}
$$

where $L$ is the quantum number for the total orbital angular momentum of the electrons and $S$ the quantum number for the total spin of the electrons. It follows from the addition of angular momenta that the quadrupole matrix elements may be nonzero only if $\left|L^{\prime}-L\right| \leqslant 2 \leqslant L^{\prime}+L$. On the other hand, the parity of the tensor $T_{q}^{(2)} \propto Y_{2 q}$ is even. Therefore, the quadrupole matrix elements may be nonzero only if $L$ and $L^{\prime}$ have the same parity. Thus, the electric quadrupole transition selection rules for $L$ and $L^{\prime}$ are $\left|L^{\prime}-L\right|=0,2$ and $L^{\prime}+L \geqslant 2$. We note that, in the special case where $L=0$ and $L^{\prime}=2$, we have $\left\langle n^{\prime}, L^{\prime}=2\left\|T^{(2)}\right\| n, L=0\right\rangle=\sqrt{2 / 3}\left\langle n^{\prime}, L^{\prime}=\right.$ $\left.2\left|R^{2}\right| n, L=0\right\rangle$.

\section{APPENDIX B: QUADRUPOLE INTERACTION OF AN ATOM WITH A PLANE-WAVE LIGHT FIELD IN FREE SPACE}

Assume that the field is a plane wave $\mathcal{E}=\mathcal{E} \boldsymbol{\varepsilon} e^{i \mathbf{k} \cdot \mathbf{x}}$ in free space, where $\mathcal{E}$ is the amplitude, $\mathbf{k}$ is the wave vector, and $\boldsymbol{\varepsilon}$ is the polarization vector. In this case, the rms Rabi frequency $\bar{\Omega}_{F F^{\prime}}^{(0)}$ is found from Eq. (8) to be

$$
\bar{\Omega}_{F F^{\prime}}^{(0) 2}=\frac{e^{2}|\mathcal{E}|^{2}}{20 \hbar^{2}}\left|\left\langle n^{\prime} F^{\prime}\left\|T^{(2)}\right\| n F\right\rangle\right|^{2} \sum_{q}\left|\sum_{i j} u_{i j}^{(q)} k_{i} \varepsilon_{j}\right|^{2} .
$$

Without loss of generality, we assume that the field propagates along the $x_{3}$ direction and is linearly polarized along the $x_{1}$ direction. Then, we have $\mathbf{k}=(0,0, k)$ and $\boldsymbol{\varepsilon}=(1,0,0)$ in the Cartesian coordinate system $\left\{x_{1}, x_{2}, x_{3}\right\}$. These expressions lead to $k_{i}=k \delta_{i, 3}$ and $\varepsilon_{j}=\delta_{j, 1}$. Then, Eq. (B1) gives

$$
\bar{\Omega}_{F F^{\prime}}^{(0) 2}=\frac{e^{2} k^{2}|\mathcal{E}|^{2}}{20 \hbar^{2}}\left|\left\langle n^{\prime} F^{\prime}\left\|T^{(2)}\right\| n F\right\rangle\right|^{2} \sum_{q}\left|u_{31}^{(q)}\right|^{2} .
$$

From Eqs. (A12), we find $\sum_{q}\left|u_{31}^{(q)}\right|^{2}=1 / 2$. Hence, we obtain

$$
\bar{\Omega}_{F F^{\prime}}^{(0) 2}=\frac{e^{2} k^{2}|\mathcal{E}|^{2}}{40 \hbar^{2}}\left|\left\langle n^{\prime} F^{\prime}\left\|T^{(2)}\right\| n F\right\rangle\right|^{2} .
$$

The oscillator strength $f_{F F^{\prime}}^{(0)}$ is related to the rms Rabi frequency $\bar{\Omega}_{F F^{\prime}}^{(0)}$ via formula (9). With the help of this formula, 
we find

$$
f_{F F^{\prime}}^{(0)}=\frac{m_{e} \omega_{0}^{3}}{20 \hbar c^{2}} \frac{\left|\left\langle n^{\prime} F^{\prime}\left\|T^{(2)}\right\| n F\right\rangle\right|^{2}}{2 F+1} .
$$

The oscillator strength $f_{J J^{\prime}}^{(0)}$ of the transition from a lower finestructure level $|n J\rangle$ to an upper fine-structure level $\left|n^{\prime} J^{\prime}\right\rangle$ of the atom in free space may be obtained by summing up $f_{F F^{\prime}}^{(0)}$ over all values of $F^{\prime}$. The result is $[6,7,20]$

$$
f_{J J^{\prime}}^{(0)}=\frac{m_{e} \omega_{0}^{3}}{20 \hbar c^{2}} \frac{\left|\left\langle n^{\prime} J^{\prime}\left\|T^{(2)}\right\| n J\right\rangle\right|^{2}}{2 J+1} .
$$

The rate $\gamma_{F^{\prime} F}$ of quadrupole spontaneous emission from an upper hyperfine-structure level $\left|n^{\prime} F^{\prime}\right\rangle$ to a lower hyperfinestructure level $|n F\rangle$ of the atom in free space is related to the oscillator strength $f_{F F^{\prime}}^{(0)}$ as

$$
\gamma_{F^{\prime} F}^{(0)}=\frac{e^{2} \omega_{0}^{2}}{2 \pi \epsilon_{0} m_{e} c^{3}} \frac{2 F+1}{2 F^{\prime}+1} f_{F F^{\prime}}^{(0)}
$$

Hence, we find

$$
\gamma_{F^{\prime} F}^{(0)}=\frac{e^{2} \omega_{0}^{5}}{40 \pi \epsilon_{0} \hbar c^{5}} \frac{\left|\left\langle n^{\prime} F^{\prime}\left\|T^{(2)}\right\| n F\right\rangle\right|^{2}}{2 F^{\prime}+1} .
$$

The rate $\gamma_{J^{\prime} J}^{(0)}$ of quadrupole spontaneous emission from an upper fine-structure level $\left|n^{\prime} J^{\prime}\right\rangle$ to a lower fine-structure level $|n J\rangle$ of the atom in free space may be obtained by summing up $\gamma_{F^{\prime} F}^{(0)}$ over all values of $F$. The result is $[6,7,20]$

$$
\gamma_{J^{\prime} J}^{(0)}=\frac{e^{2} \omega_{0}^{5}}{40 \pi \epsilon_{0} \hbar c^{5}} \frac{\left|\left\langle n^{\prime} J^{\prime}\left\|T^{(2)}\right\| n J\right\rangle\right|^{2}}{2 J^{\prime}+1} .
$$

We have the relation

$$
\gamma_{J^{\prime} J}^{(0)}=\frac{e^{2} \omega_{0}^{2}}{2 \pi \epsilon_{0} m_{e} c^{3}} \frac{2 J+1}{2 J^{\prime}+1} f_{J J^{\prime}}^{(0)}
$$

It follows from Eq. (A15) that the relations between $\gamma_{F^{\prime} F}^{(0)}$ and $\gamma_{J^{\prime} J}^{(0)}$ and between $f_{F F^{\prime}}^{(0)}$ and $f_{J J^{\prime}}^{(0)}$ are $[18,19,38]$

$$
\begin{aligned}
& \gamma_{F^{\prime} F}^{(0)}=(2 F+1)\left(2 J^{\prime}+1\right)\left\{\begin{array}{ccc}
F^{\prime} & 2 & F \\
J & I & J^{\prime}
\end{array}\right\}^{2} \gamma_{J^{\prime} J}^{(0)}, \\
& f_{F F^{\prime}}^{(0)}=\left(2 F^{\prime}+1\right)(2 J+1)\left\{\begin{array}{lll}
F^{\prime} & 2 & F \\
J & I & J^{\prime}
\end{array}\right\}^{2} f_{J J^{\prime}}^{(0)}
\end{aligned}
$$

\section{APPENDIX C: GUIDED MODES OF A STEP-INDEX FIBER}

Consider the model of a step-index fiber that is a dielectric cylinder of radius $a$ and refractive index $n_{1}$ and is surrounded by an infinite background medium of refractive index $n_{2}$, where $n_{2}<n_{1}$. For a guided light field of frequency $\omega$ (freespace wavelength $\lambda=2 \pi c / \omega$ and free-space wave number $k=\omega / c)$, the propagation constant $\beta$ is determined by the fiber eigenvalue equation [40]

$$
\begin{aligned}
& {\left[\frac{J_{l}^{\prime}(h a)}{h a J_{l}(h a)}+\frac{K_{l}^{\prime}(q a)}{q a K_{l}(q a)}\right]\left[\frac{n_{1}^{2} J_{l}^{\prime}(h a)}{h a J_{l}(h a)}+\frac{n_{2}^{2} K_{l}^{\prime}(q a)}{q a K_{l}(q a)}\right]} \\
& \quad=l^{2}\left(\frac{1}{h^{2} a^{2}}+\frac{1}{q^{2} a^{2}}\right)^{2} \frac{\beta^{2}}{k^{2}} .
\end{aligned}
$$

Here, we have introduced the parameters $h=\left(n_{1}^{2} k^{2}-\beta^{2}\right)^{1 / 2}$ and $q=\left(\beta^{2}-n_{2}^{2} k^{2}\right)^{1 / 2}$, which characterize the scales of the spatial variations of the field inside and outside the fiber, respectively. The integer index $l=0,1,2, \ldots$ is the azimuthal mode order, which determines the helical phase front and the associated phase gradient in the fiber transverse plane. The notations $J_{l}$ and $K_{l}$ stand for the Bessel functions of the first kind and the modified Bessel functions of the second kind, respectively. The notations $J_{l}^{\prime}(x)$ and $K_{l}^{\prime}(x)$ stand for the derivatives of $J_{l}(x)$ and $K_{l}(x)$ with respect to the argument $x$. We note that the fiber eigenvalue equation $(\mathrm{C} 1)$ remains the same when we replace $\beta$ by $-\beta$ or $l$ by $-l$.

For $l \geqslant 1$, the eigenvalue equation (C1) leads to hybrid HE and $\mathrm{EH}$ modes [40]. The eigenvalue equation is given, for $\mathrm{HE}$ modes, as

$$
\frac{J_{l-1}(h a)}{h a J_{l}(h a)}=-\frac{n_{1}^{2}+n_{2}^{2}}{2 n_{1}^{2}} \frac{K_{l}^{\prime}(q a)}{q a K_{l}(q a)}+\frac{l}{h^{2} a^{2}}-\mathcal{R}
$$

and, for EH modes, as

$$
\frac{J_{l-1}(h a)}{h a J_{l}(h a)}=-\frac{n_{1}^{2}+n_{2}^{2}}{2 n_{1}^{2}} \frac{K_{l}^{\prime}(q a)}{q a K_{l}(q a)}+\frac{l}{h^{2} a^{2}}+\mathcal{R} .
$$

Here, we have introduced the notation

$$
\begin{aligned}
\mathcal{R}= & {\left[\left(\frac{n_{1}^{2}-n_{2}^{2}}{2 n_{1}^{2}}\right)^{2}\left(\frac{K_{l}^{\prime}(q a)}{q a K_{l}(q a)}\right)^{2}\right.} \\
& \left.+\left(\frac{l \beta}{n_{1} k}\right)^{2}\left(\frac{1}{q^{2} a^{2}}+\frac{1}{h^{2} a^{2}}\right)^{2}\right]^{1 / 2} .
\end{aligned}
$$

We label $\mathrm{HE}$ and $\mathrm{EH}$ modes as $\mathrm{HE}_{l m}$ and $\mathrm{EH}_{l m}$, respectively, where $l=1,2, \ldots$ and $m=1,2, \ldots$ are the azimuthal and radial mode orders, respectively. Here, the radial mode order $m$ implies that the $\mathrm{HE}_{l m}$ or $\mathrm{EH}_{l m}$ mode is the $m$ th solution to the corresponding eigenvalue equation (C2) or (C3), respectively.

For $l=0$, the eigenvalue equation ( $\mathrm{C} 1$ ) leads to transverse electric modes TE and transverse magnetic modes TM [40]. The eigenvalue equation is given, for TE modes, as

$$
\frac{J_{1}(h a)}{h a J_{0}(h a)}=-\frac{K_{1}(q a)}{q a K_{0}(q a)}
$$

and, for TM modes, as

$$
\frac{J_{1}(h a)}{h a J_{0}(h a)}=-\frac{n_{2}^{2}}{n_{1}^{2}} \frac{K_{1}(q a)}{q a K_{0}(q a)} .
$$

We label TE and TM modes as $\mathrm{TE}_{0 m}$ and $\mathrm{TM}_{0 m}$, respectively, where $m=1,2, \ldots$ is the radial mode order. The subscript 0 implies that the azimuthal mode order of TE and TM modes is $l=0$. The radial mode order $m$ implies that the $\mathrm{TE}_{0 m}$ or $\mathrm{TM}_{0 m}$ mode is the $m$ th solution to the corresponding eigenvalue equation (C5) or (C6), respectively.

According to Ref. [40], the fiber size parameter $V$ is defined as $V=k a \sqrt{n_{1}^{2}-n_{2}^{2}}$. The cutoff values $V_{c}$ for $\mathrm{HE}_{1 m}$ modes are determined as solutions to the equation $J_{1}\left(V_{c}\right)=0$. For $\mathrm{HE}_{l m}$ modes with $l=2,3, \ldots$, the cutoff values are obtained as nonzero solutions to the equation $\left(n_{1}^{2} / n_{2}^{2}+1\right)(l-$ 1) $J_{l-1}\left(V_{c}\right)=V_{c} J_{l}\left(V_{c}\right)$. The cutoff values $V_{c}$ for $\mathrm{EH}_{l m}$ modes, where $l=1,2, \ldots$, are determined as nonzero solutions to the equation $J_{l}\left(V_{c}\right)=0$. For $\mathrm{TE}_{0 m}$ and $\mathrm{TM}_{0 m}$ modes, the cutoff values $V_{c}$ are obtained as solutions to the equation $J_{0}\left(V_{c}\right)=0$. 
The electric component of the field can be presented in the form

$$
\mathbf{E}=\frac{1}{2} \mathcal{E} e^{-i \omega t}+\text { c.c. }
$$

where $\mathcal{E}$ is the amplitude. For a guided mode with a propagation constant $\beta$ and an azimuthal mode order $l$, we can write

$$
\mathcal{E}=\mathbf{e} e^{i \beta z+i l \varphi}
$$

where $\mathbf{e}$ is the mode profile function. In order to construct the profile functions of a complete set of guided modes, we allow $\beta$ and $l$ in Eq. (C8) to take not only positive but also negative values. We decompose the vectorial function $\mathbf{e}$ into the radial, azimuthal, and axial components denoted by the subscripts $r$, $\varphi$, and $z$, respectively. We summarize the expressions for the mode functions of quasicircularly polarized hybrid modes, TE modes, and TM modes in the following [40].

\section{Quasicircularly polarized hybrid modes}

We consider quasicircularly polarized hybrid modes $N=$ $\mathrm{HE}_{l m}$ or $\mathrm{EH}_{l m}$. It is convenient to introduce the parameter

$$
s=l\left(\frac{1}{h^{2} a^{2}}+\frac{1}{q^{2} a^{2}}\right)\left[\frac{J_{l}^{\prime}(h a)}{h a J_{l}(h a)}+\frac{K_{l}^{\prime}(q a)}{q a K_{l}(q a)}\right]^{-1} .
$$

Then, we find, for $r<a$,

$$
\begin{aligned}
e_{r} & =i A \frac{\beta}{2 h}\left[(1-s) J_{l-1}(h r)-(1+s) J_{l+1}(h r)\right], \\
e_{\varphi} & =-A \frac{\beta}{2 h}\left[(1-s) J_{l-1}(h r)+(1+s) J_{l+1}(h r)\right], \\
e_{z} & =A J_{l}(h r),
\end{aligned}
$$

and, for $r>a$,

$$
\begin{aligned}
e_{r} & =i A \frac{\beta}{2 q} \frac{J_{l}(h a)}{K_{l}(q a)}\left[(1-s) K_{l-1}(q r)+(1+s) K_{l+1}(q r)\right], \\
e_{\varphi} & =-A \frac{\beta}{2 q} \frac{J_{l}(h a)}{K_{l}(q a)}\left[(1-s) K_{l-1}(q r)-(1+s) K_{l+1}(q r)\right], \\
e_{z} & =A \frac{J_{l}(h a)}{K_{l}(q a)} K_{l}(q r) .
\end{aligned}
$$

Here, the parameter $A$ is a constant that can be determined from the propagating power of the field.

\section{TE modes}

We consider transverse electric modes $N=\mathrm{TE}_{0 m}$. For $r<a$, we have

$$
e_{r}=0, \quad e_{\varphi}=i \frac{\omega \mu_{0}}{h} A J_{1}(h r), \quad e_{z}=0 .
$$

For $r>a$, we have

$$
e_{r}=0, \quad e_{\varphi}=-i \frac{\omega \mu_{0}}{q} \frac{J_{0}(h a)}{K_{0}(q a)} A K_{1}(q r), \quad e_{z}=0
$$

\section{TM modes}

We consider transverse magnetic modes $N=\mathrm{TM}_{0 m}$. For $r<a$, we have

$$
e_{r}=-i \frac{\beta}{h} A J_{1}(h r), \quad e_{\varphi}=0, \quad e_{z}=A J_{0}(h r) .
$$

For $r>a$, we have

$$
\begin{aligned}
& e_{r}=i \frac{\beta}{q} \frac{J_{0}(h a)}{K_{0}(q a)} A K_{1}(q r), \\
& e_{\varphi}=0, \\
& e_{z}=\frac{J_{0}(h a)}{K_{0}(q a)} A K_{0}(q r) .
\end{aligned}
$$

[1] W. Demtröder, Atoms, Molecules and Photons (Springer, Berlin, 2010).

[2] A. D. Ludlow, M. M. Boyd, J. Ye, E. Peik, and P. O. Schmidt, Rev. Mod. Phys. 87, 637 (2015).

[3] H. Häffner, C. F. Roos, and R. Blatt, Phys. Rep. 469, 155 (2008).

[4] K. Niemax, J. Quant. Spectrosc. Radiat. Transf. 17, 747 (1977).

[5] J. Nilsen and J. Marling, J. Quant. Spectrosc. Radiat. Transf. 20, 327 (1978).

[6] H. S. Freedhoff, J. Chem. Phys. 54, 1618 (1971); J. Phys. B 22, 435 (1989).

[7] D. F. V. James, Appl. Phys. B 66, 181 (1998).

[8] C. T. Schmiegelow, J. Schulz, H. Kaufmann, T. Ruster, U. G. Poschinger, and F. Schmidt-Kaler, Nat. Commun. 7, 12998 (2016).

[9] R. Jáuregui, Phys. Rev. A 91, 043842 (2015).

[10] A. Afanasev, C. E. Carlson, and A. Mukherjee, J. Opt. 18, 074013 (2016).

[11] A. A. Peshkov, D. Seipt, A. Surzhykov, and S. Fritzsche, Phys. Rev. A 96, 023407 (2017).
[12] A. Afanasev, C. E. Carlson, and M. Solyanik, J. Opt. 19, 105401 (2017).

[13] A. Afanasev, C. E. Carlson, C. T. Schmiegelow, J. Schulz, F. Schmidt-Kaler, and M. Solyanik, arXiv:1709.05571.

[14] P. K. Mondal, B. Deb, and S. Majumder, Phys. Rev. A 89, 063418 (2014).

[15] E. A. Chan, S. A. Aljunid, N. I. Zheludev, D. Wilkowski, and M. Ducloy, Opt. Lett. 41, 2005 (2016).

[16] M. Germann, X. Tong, and S. Willitsch, Nat. Phys. 10, 820 (2014).

[17] D. Tong, S. M. Farooqi, E. G. M. van Kempen, Z. Pavlovic, J. Stanojevic, R. Côté, E. E. Eyler, and P. L. Gould, Phys. Rev. A 79, 052509 (2009).

[18] S. Tojo, M. Hasuo, and T. Fujimoto, Phys. Rev. Lett. 92, 053001 (2004)

[19] S. Tojo, T. Fujimoto, and M. Hasuo, Phys. Rev. A 71, 012507 (2005).

[20] S. Tojo and M. Hasuo, Phys. Rev. A 71, 012508 (2005).

[21] V. V. Klimov and V. S. Letokhov, Phys. Rev. A 54, 4408 (1996).

[22] V. V. Klimov and M. Ducloy, Phys. Rev. A 62, 043818 (2000). 
[23] A. M. Kern and O. J. F. Martin, Phys. Rev. A 85, 022501 (2012).

[24] K. Shibata, S. Tojo, and D. Bloch, Opt. Express 25, 9476 (2017).

[25] L. Tong, R. R. Gattass, J. B. Ashcom, S. He, J. Lou, M. Shen, I. Maxwell, and E. Mazur, Nature (London) 426, 816 (2003).

[26] For a review, see T. Nieddu, V. Gokhroo, and S. Nic Chormaic, J. Opt. 18, 053001 (2016).

[27] For a more recent review, see P. Solano, J. A. Grover, J. E. Homan, S. Ravets, F. K. Fatemi, L. A. Orozco, and S. L. Rolston, Adv. At. Mol. Opt. Phys. 66, 439 (2017).

[28] L. Tong, J. Lou, and E. Mazur, Opt. Express 12, 1025 (2004).

[29] Fam Le Kien, J. Q. Liang, K. Hakuta, and V. I. Balykin, Opt. Commun. 242, 445 (2004).

[30] V. I. Balykin, K. Hakuta, Fam Le Kien, J. Q. Liang, and M. Morinaga, Phys. Rev. A 70, 011401(R) (2004); Fam Le Kien, V. I. Balykin, and K. Hakuta, ibid. 70, 063403 (2004).

[31] E. Vetsch, D. Reitz, G. Sagué, R. Schmidt, S. T. Dawkins, and A. Rauschenbeutel, Phys. Rev. Lett. 104, 203603 (2010).

[32] A. Goban, K. S. Choi, D. J. Alton, D. Ding, C. Lacroûte, M. Pototschnig, T. Thiele, N. P. Stern, and H. J. Kimble, Phys. Rev. Lett. 109, 033603 (2012).

[33] R. Kumar, V. Gokhroo, K. Deasy, A. Maimaiti, M. C. Frawley, C. Phelan, and S. Nic Chormaic, New. J. Phys. 17, 013026 (2015).

[34] Fam Le Kien, Th. Busch, V. G. Truong, and S. Nic Chormaic, Phys. Rev. A 96, 023835 (2017).

[35] Fam Le Kien, Th. Busch, V. G. Truong, and S. Nic Chormaic, Commun. Phys. 27, 23 (2017).

[36] See, for example, J. D. Jackson, Classical Electrodynamics, 3rd ed. (Wiley, New York, 1999).

[37] B. W. Shore, The Theory of Coherent Atomic Excitation (Wiley, New York, 1990).

[38] Note that Eq. (4) of Ref. [18] and Eqs. (4) and (7) of Ref. [19] are not accurate. The factor $2 F+1$ in these equations must be replaced by the factor $2 J+1$.

[39] Fam Le Kien and K. Hakuta, Phys. Rev. A 75, 013423 (2007).

[40] See, for example, D. Marcuse, Light Transmission Optics (Krieger, Malabar, FL, 1989); A. W. Snyder and J. D. Love, Optical Waveguide Theory (Chapman and Hall, New York, 1983); K. Okamoto, Fundamentals of Optical Waveguides (Elsevier, New York, 2006).

[41] A. V. Dooghin, N. D. Kundikova, V. S. Liberman, and B. Y. Zeldovich, Phys. Rev. A 45, 8204 (1992); V. S. Liberman and B. Y. Zeldovich, ibid. 46, 5199 (1992); M. Y. Darsht, B. Y.
Zeldovich, I. V. Kataevskaya, and N. D. Kundikova, Zh. Eksp. Teor. Phys. 107, 1464 (1995) [JETP 80, 817 (1995)].

[42] K. Y. Bliokh, A. Aiello, and M. A. Alonso, in The Angular Momentum of Light, edited by D. L. Andrews and M. Babiker (Cambridge University Press, New York, 2012), p. 174.

[43] K. Y. Bliokh, J. Dressel, and F. Nori, New J. Phys. 16, 093037 (2014).

[44] K. Y. Bliokh, A. Y. Bekshaev, and F. Nori, Nat. Commun. 5, 3300 (2014).

[45] K. Y. Bliokh and F. Nori, Phys. Rep. 592, 1 (2015).

[46] A. Aiello, P. Banzer, M. Neugebauer, and G. Leuchs, Nat. Photon. 9, 789 (2015).

[47] K. Y. Bliokh, F. J. Rodriguez-Fortuño, F. Nori, and A. V. Zayats, Nat. Photon. 9, 796 (2015).

[48] Fam Le Kien and A. Rauschenbeutel, Phys. Rev. A 90, 023805 (2014).

[49] J. Petersen, J. Volz, and A. Rauschenbeutel, Science 346, 67 (2014).

[50] R. Mitsch, C. Sayrin, B. Albrecht, P. Schneeweiss, and A. Rauschenbeutel, Nat. Commun. 5, 5713 (2014).

[51] Fam Le Kien and A. Rauschenbeutel, Phys. Rev. A 90, 063816 (2014).

[52] C. Sayrin, C. Junge, R. Mitsch, B. Albrecht, D. O'Shea, P. Schneeweiss, J. Volz, and A. Rauschenbeutel, Phys. Rev. X 5, 041036 (2015).

[53] P. Lodahl, S. Mahmoodian, S. Stobbe, P. Schneeweiss, J. Volz, A. Rauschenbeutel, H. Pichler, and P. Zoller, Nature (London) 541, 473 (2017).

[54] Fam Le Kien, S. S. S. Hejazi, Th. Busch, V. G. Truong, and S. Nic Chormaic, Phys. Rev. A 96, 043859 (2017).

[55] S. Okaba, T. Takano, F. Benabid, T. Bradley, L. Vincetti, Z. Maizelis, V. Yampol'skii, F. Nori, and H. Katori, Nat. Commun. 5, 4096 (2014).

[56] T. Chanelière, D. N. Matsukevich, S. D. Jenkins, T. A. B. Kennedy, M. S. Chapman, and A. Kuzmich, Phys. Rev. Lett. 96, 093604 (2006).

[57] R. Roy, P. C. Condylis, Y. J. Johnathan, and B. Hessmo, Opt. Express 25, 7960 (2017).

[58] D. A. Varshalovich, A. N. Moskalev, and V. K. Khersonskii, Quantum Theory of Angular Momentum (World Scientific, Singapore, 2008); A. R. Edmonds, Angular Momentum in Quantum Mechanics (Princeton University Press, Princeton, NJ, 1974). 